\title{
The Effect of Lithium Drug on Binding Affinities of Glycogen Synthase Kinase-3 beta to its Network Partners: a New Computational Approach
}

Maryam Rouhani ${ }^{1, *}$, Hamid Hadi-Alijanvand ${ }^{1}$

${ }^{1}$ Department of Biological Sciences, Institute for Advanced Studies in Basic Sciences (IASBS), Zanjan 45137-66731, Iran

* Corresponding Author:

Maryam Rouhani,

Email: rouhani@iasbs.ac.ir

Tel: +98-24-33153314

Fax: $+98-24-33153340$

ORCID: 0000-0003-2094-6568

Address: Department of Biological Sciences, Institute for Advanced Studies in Basic Sciences (IASBS), Zanjan, 45137-66731, Iran. 


\section{Supplementary Information}

Figure S1. The sampled temperature and potential.

Figure S2. Sampling period for each temperature bin in AT-MD simulations.

Figure S3. Structural descriptors along AT-MD simulation time line.

Figure S4. RMSD along AT-MD simulation time line.

Figure S5. The effects of ions on structural fluctuations of protein.

Figure S6. Changes in secondary structures over AT-MD simulation.

Figure S7. System-dependent and protein-dependent metrics for different segments of AT-MD simulations in the presence of $\mathrm{NaCl}$.

Figure S8. System -dependent and protein-dependent metrics are computed for different segments of AT-MD simulations in the presence of $\mathrm{LiCl}$.

Figures S9, S10. The trajectories of affinity values.

Figure S11. The effect of $\mathrm{LiCl}$ on the affinity of GSK-3 $\beta$ to its partners.

Movies S1a, S1b. Dynamics of GSK-3 $\beta$ in the presence of LiCl.

Movies S2a, S2b. Dynamics of GSK-3 $\beta$ in the presence of $\mathrm{NaCl}$.

Table S1. Comparison of GSK-3 $\beta$ secondary structures in the presence of sodium and lithium ions.

Table S2. Comparison of GSK-3 $\beta$ affinities towards its partners in the presence of sodium and lithium ions in the three sets of AT-MD data.

Table S3. Comparison of $z$ score values in different segments of MD simulations for set 1 data series.

Table S4. Number of GSK-3 $\beta$ PBPs with lower, equal, and higher affinities in the presence of $\mathrm{LiCl}$ than those in the presence of $\mathrm{NaCl}$ in each pathway. 

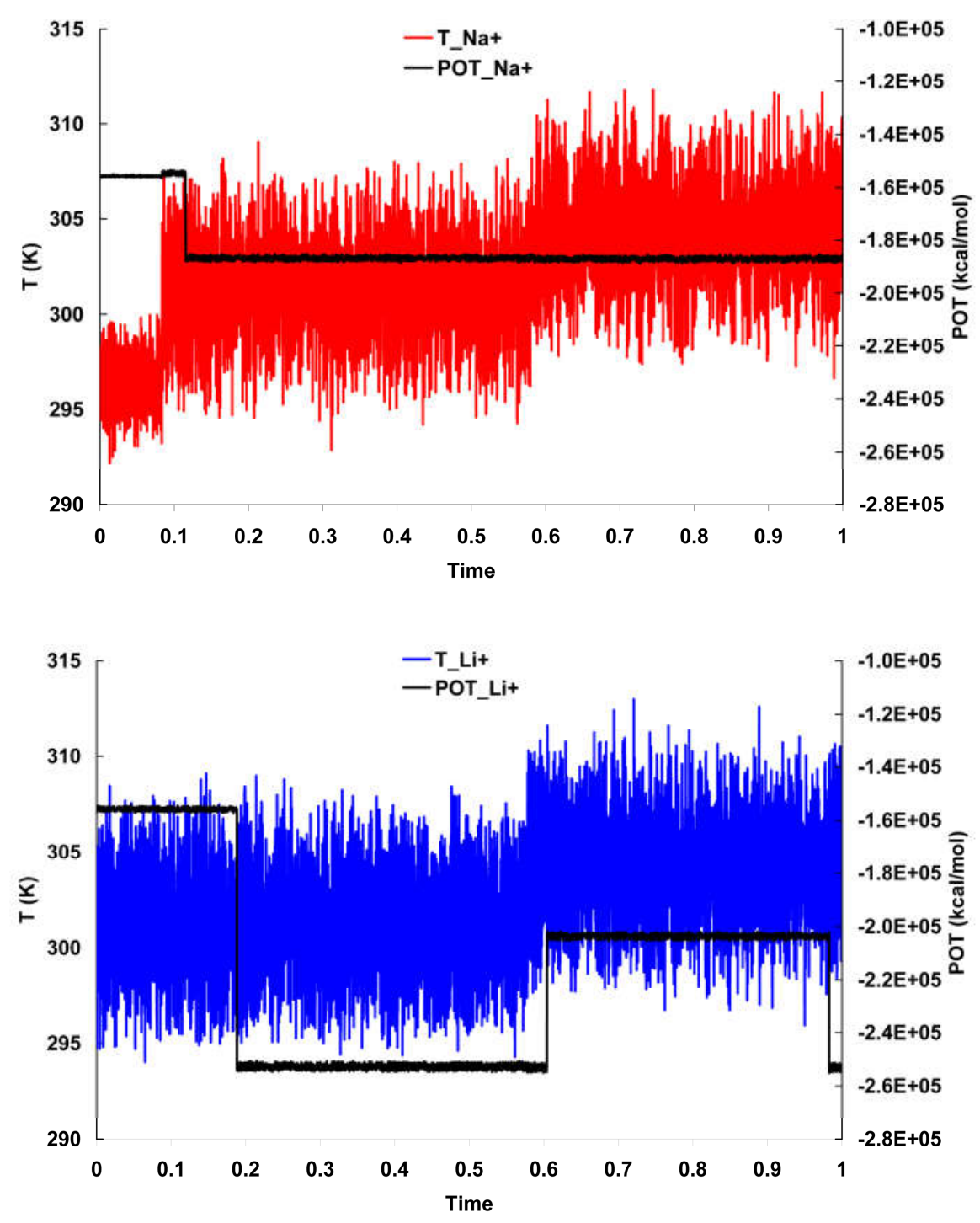

Figure S1a. The state of temperature and potential energy variations is depicted along adaptive tempering molecular dynamics simulations. On the basis of system potential energy, adaptive tempering changes the Langevin thermostat temperature randomly from 300 to $310 \mathrm{~K}$. Variations in system temperature in the presence of lithium are depicted by a blue line and for sodium by a red line. Left vertical axis denotes temperature in Kelvin and right vertical axis presents potential energy (POT) in $\mathrm{kcal} / \mathrm{mol}$ of the system. X-axis denotes relative AT-MD simulation time line. 1 indicates $155 \mathrm{~ns}$ for $\mathrm{NaCl}$ - and $225 \mathrm{~ns}$ for LiCl-containing simulations. 
GSK3b + NaCl

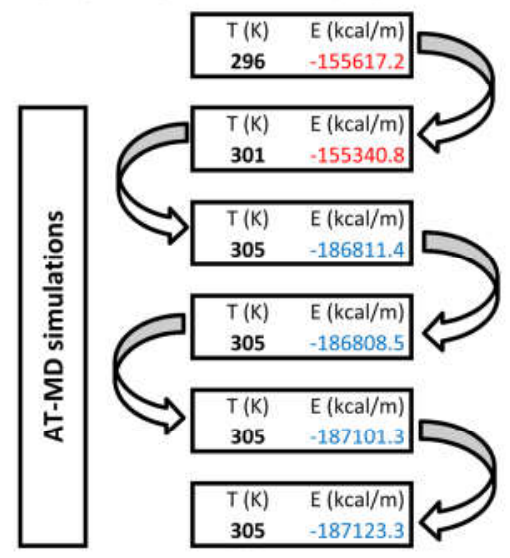

GSK3b + LiCl

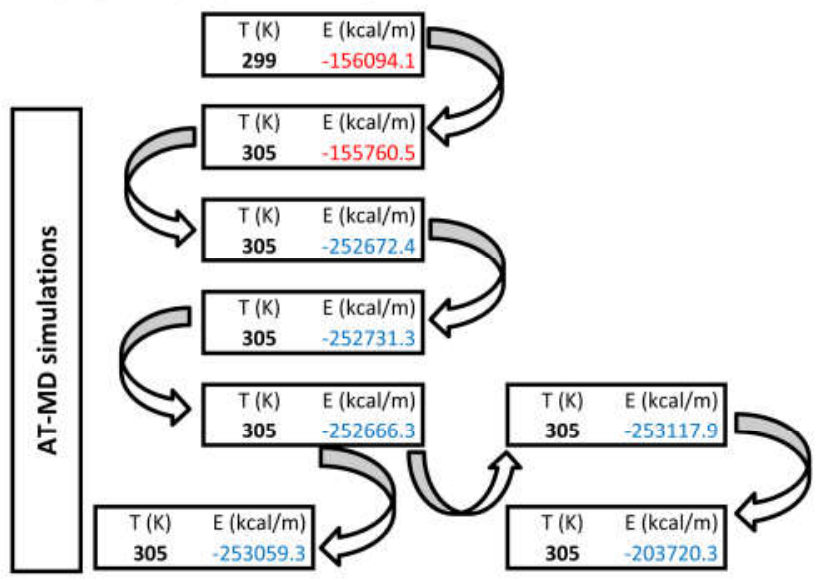

Figure S1b. Here, the mean potential energy and mean temperature for each AT-MD segment is presented. After performing regular MD simulations, we performed different segments of ATMD simulations. It is declared in scheme that GSK-3 $\beta$ experiences new basins after the first segment of AT-MD (blue printed mean potential). It should be hallmarked that GSK-3 $\beta$ experiences new potential wells in the presence of $\mathrm{LiCl}$ while the mean temperature of the segment is similar to that in the presence of $\mathrm{NaCl}$. 


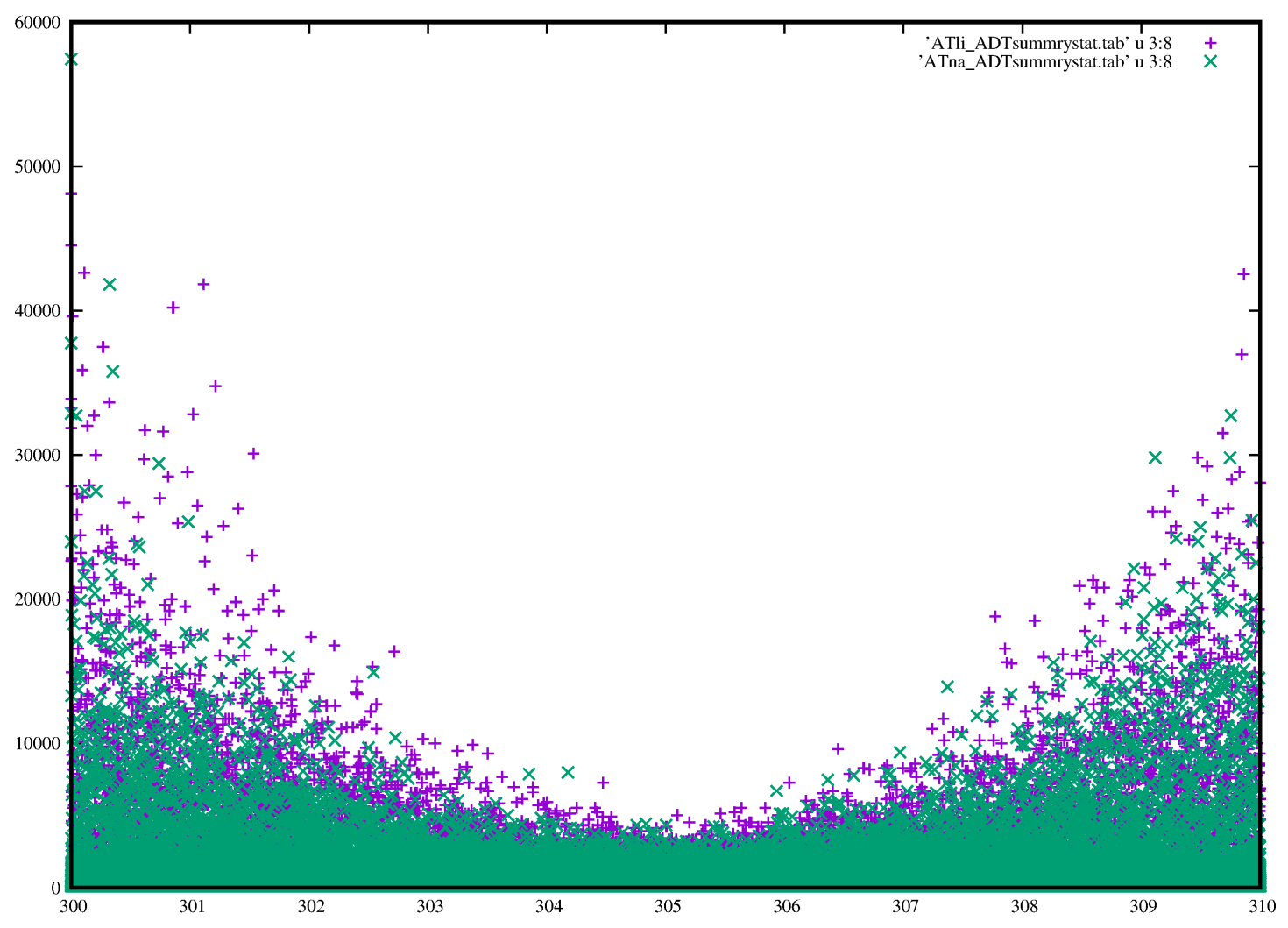

Figure S2. Sampling period for each temperature bin in AT-MD simulations.

In AT-MD simulations, temperature variations are introduced to the Langevin thermostat to overcome the potential hill ahead of structure sampling. We set the temperature range from 300 to $310 \mathrm{~K}$. The algorithm selects a temperature value from the defined range. Before changing the system temperature on the basis of potential energy history, GSK-3 $\beta$ lives in a constant temperature. The presented graph indicates the life span of GSK-3 $\beta$ in each temperature before jumping to a different temperature. $\mathrm{X}$-axis denotes temperature bins in Kelvin. Vertical axis represents the time span in fs. 

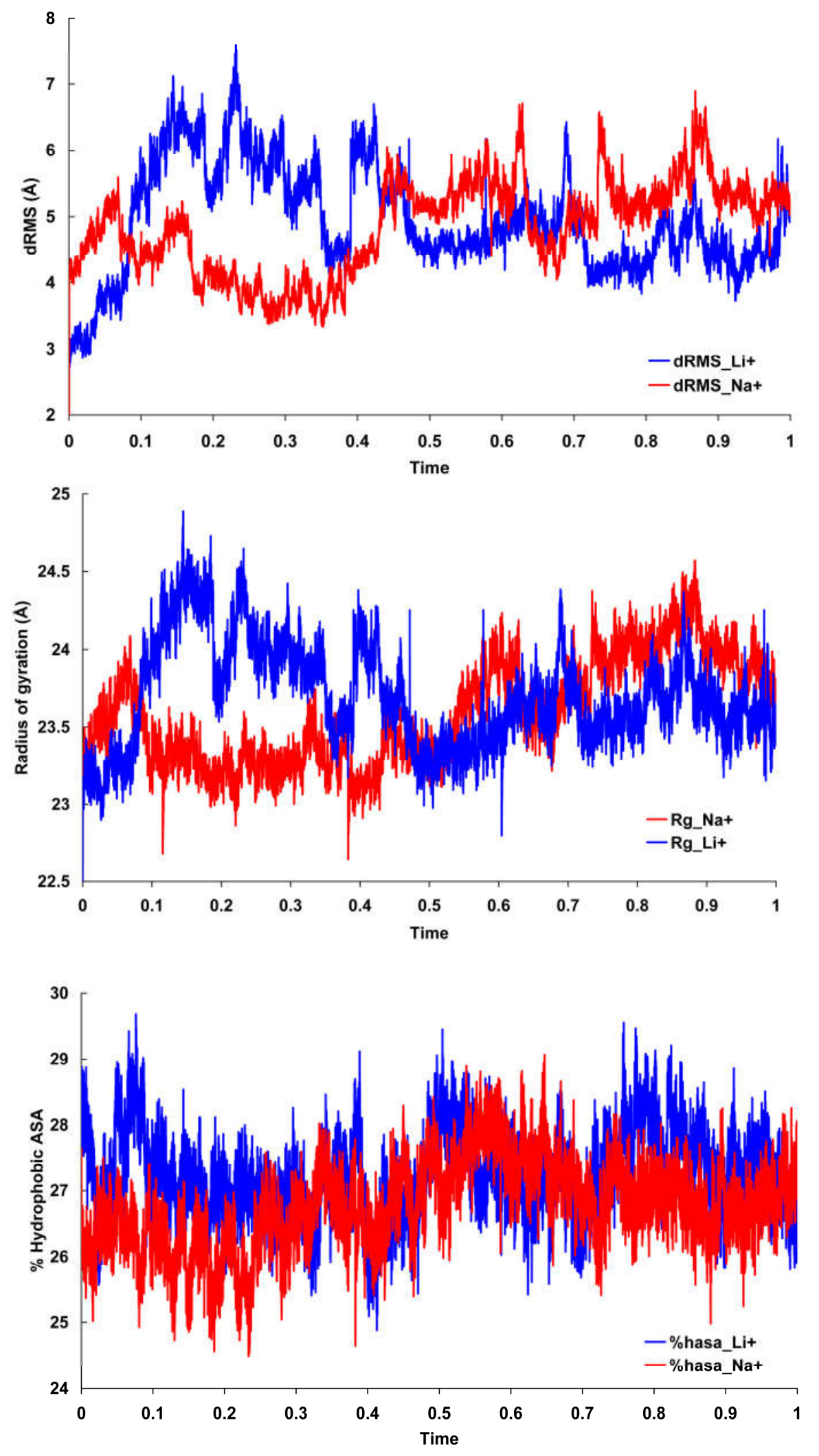

Figure S3. Structural descriptors along AT-MD simulation time line. 
The variations in internal coordinates of the GSK-3 $\beta$ along AT-MD simulation is represented in upper panel as dRMS in angstrom. The changes in the size of protein by computing radius of gyration $(\mathrm{Rg})$ in angstrom are declared in middle panel. Lower panel presents variations in percent of accessible hydrophobic surface area as a function of AT-MD simulation time line. Xaxis denotes relative AT-MD simulation time line. The properties of the systems that contain sodium are represented by red line and for lithium by blue line. 

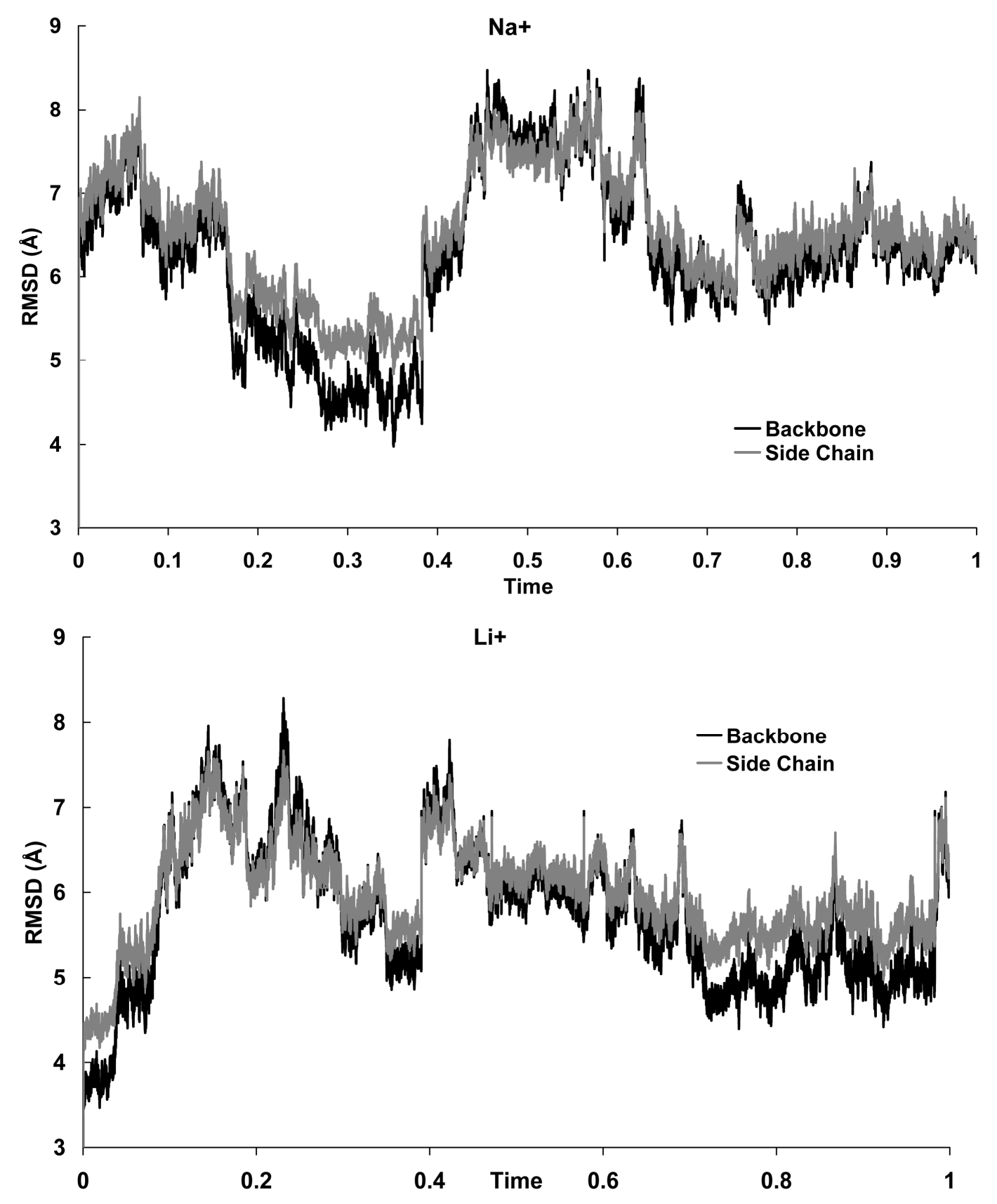

Figure S4. RMSD along AT-MD simulation time line.

We compute RMSD of each AT-MD-generated structure in regard to the starting structure of the first regular MD. Upper panel presents the RMSD values (in angstrom) for GSK-3 $\beta$ in the presence of $\mathrm{NaCl}$ and lower panel shows same in the presence of LiCl. The RMSD of backbone atoms is denoted by black line and the RMSD for side chain atoms is represented by gray line. $\mathrm{X}$-axis denotes the relative AT-MD simulation time line. 


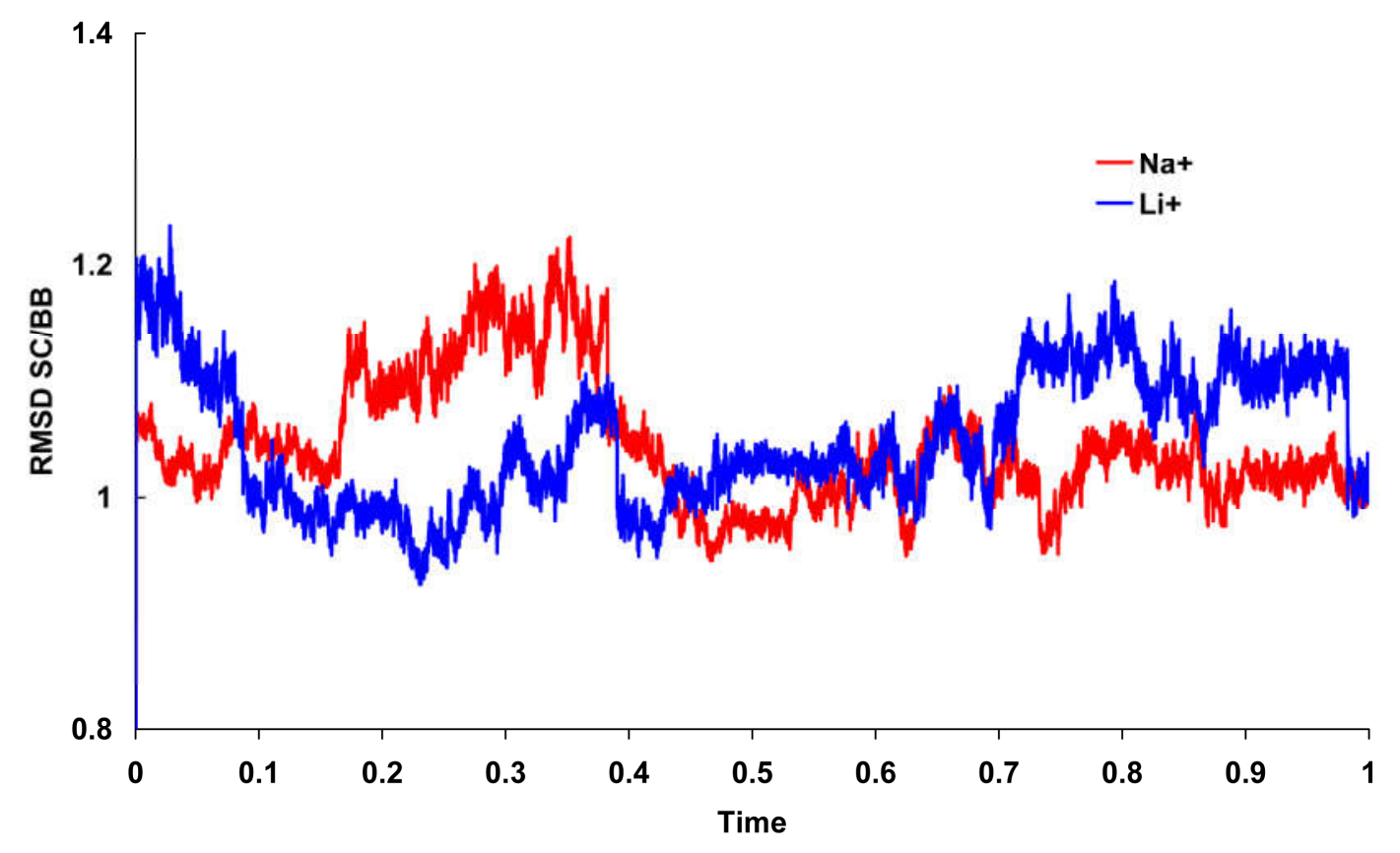

Figure S5. The effects of ions on structural fluctuations of protein.

The dominancy of side chain or backbone in GSK-3 $\beta$ structural fluctuations is presented by computing the ratio between RMSD of side chain (SC) to the RMSD of the corresponding backbone (BB) atoms for every frame along AT-MD simulation. Red line presents the effect of sodium and blue one represents the effect of lithium ion. X-axis denotes the relative AT-MD simulation time line. 


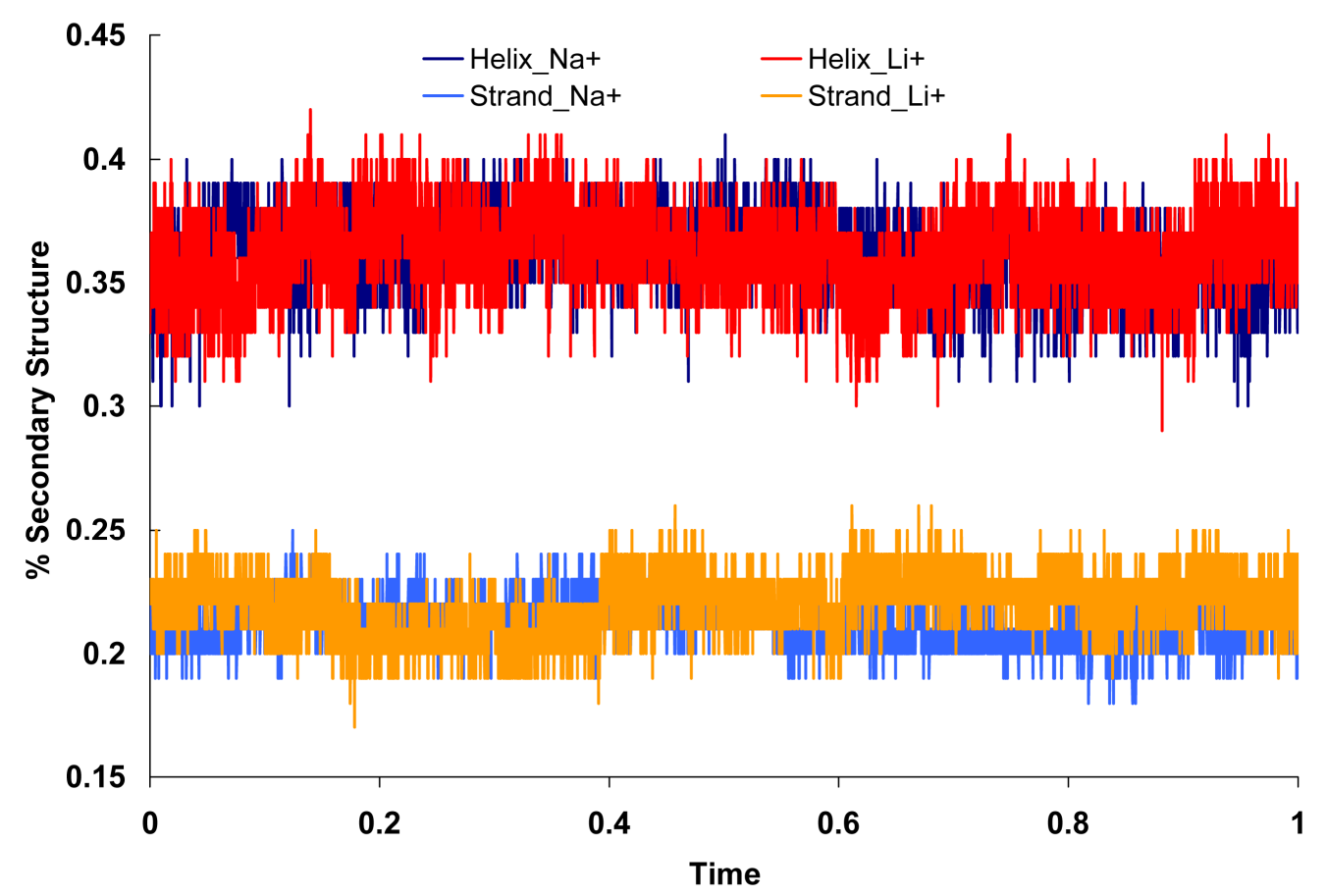

Figure S6. Changes in secondary structures over AT-MD simulation.

The DSSP-like secondary structure content of every frame is computed by WORDOM software over AT-MD simulation time. 

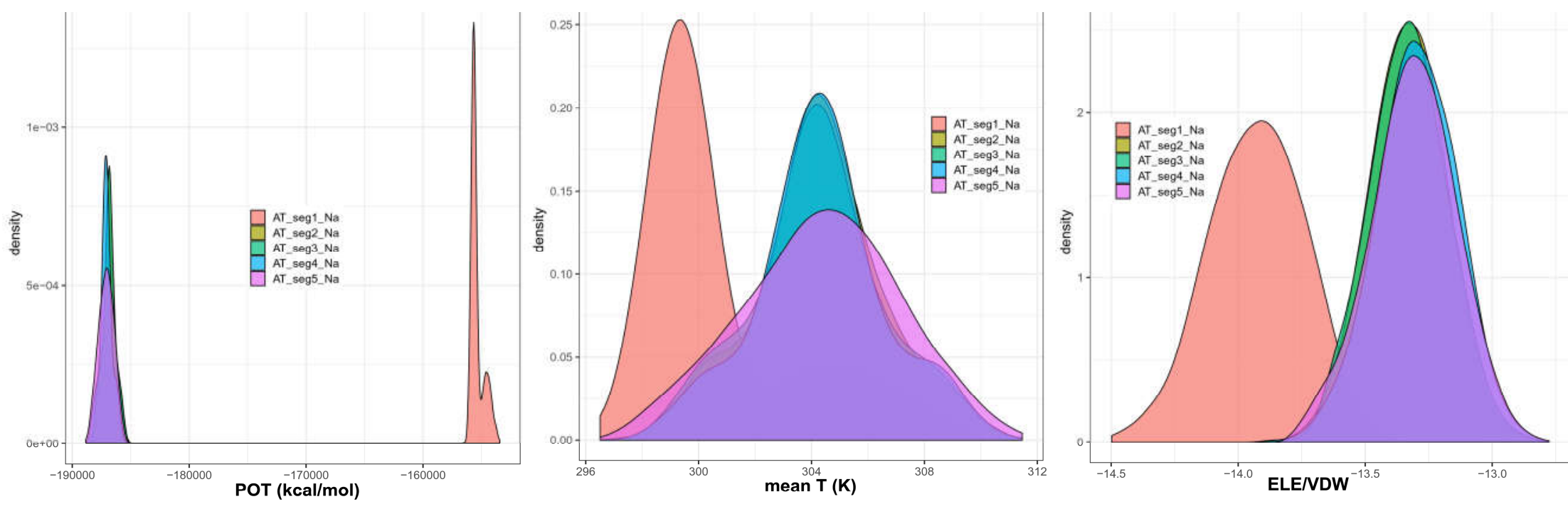

के
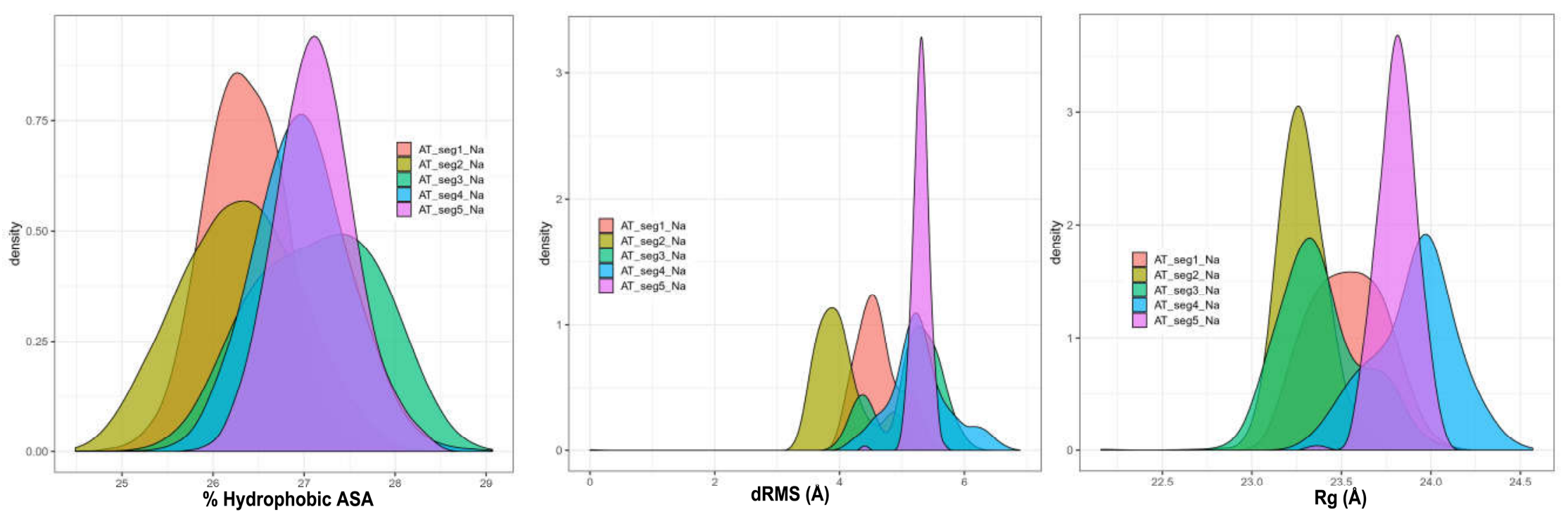

홈

Figure S7. System-dependent and protein-dependent metrics for different segments of AT-MD simulations in the presence of NaCl. 
The distribution of potential energy, mean system energy, and the ratio of electrostatic potential to VdW potential are presented for 5 segments of AT-MD simulations as system-dependent metrics. The distribution of protein size is measured by computing radius of gyration ( $\mathrm{Rg}$ ), its surface property by computing percent of hydrophobic accessible surface area (\%Hydrophobic ASA), and its global structure deviation from the ITASSER-generated model by computing dRMS. As each segment started with different structure and seed number, the start point of each segment is unique. Segments 2 to 5 experience similar general system and protein properties. 

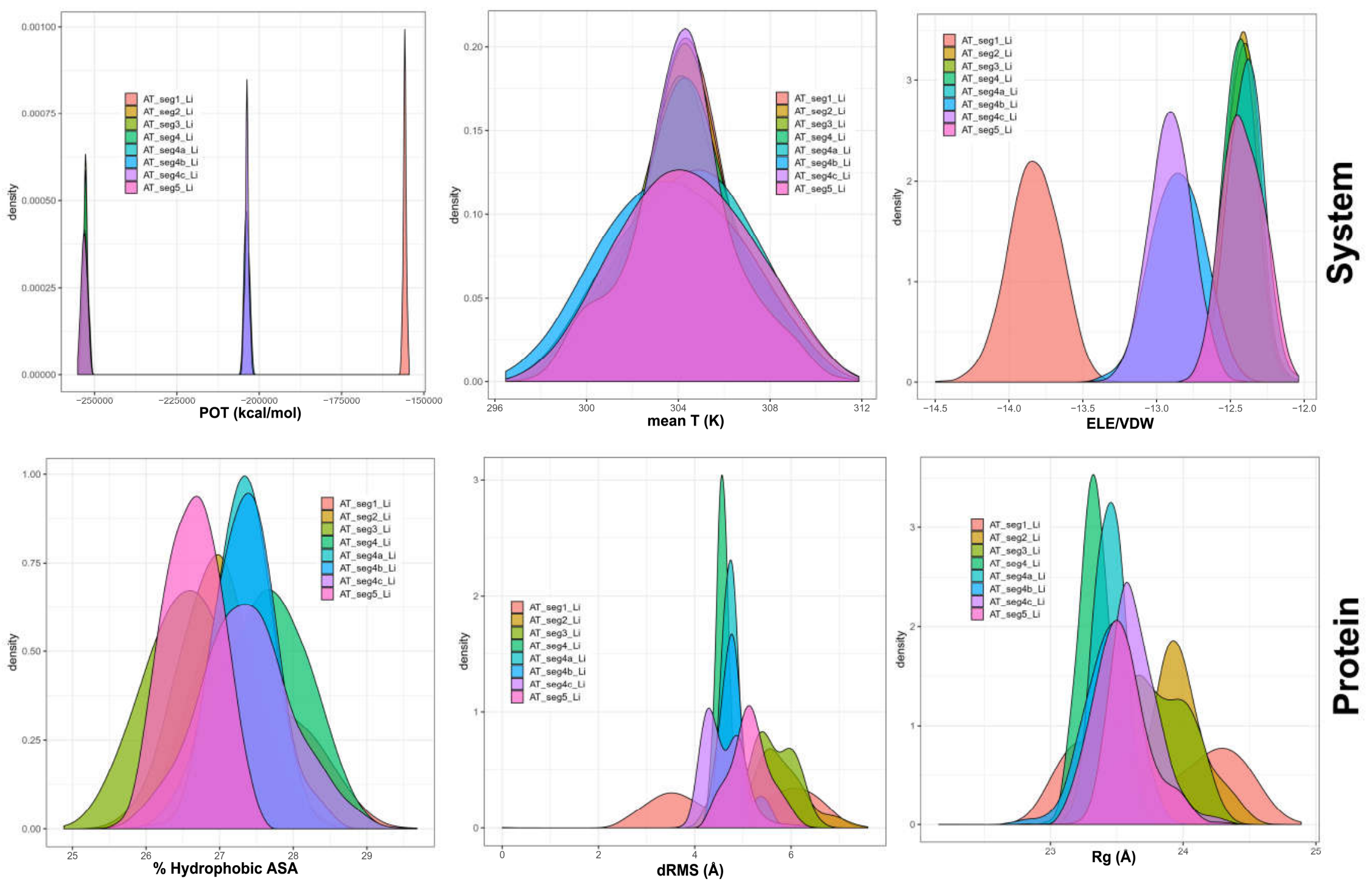

Figure S8. System -dependent and protein-dependent metrics are computed for different segments of AT-MD simulations in the presence of $\mathrm{LiCl}$. 
The distribution of potential energy, mean system energy and the ration of electric electrostatic potential to VdW potential are presented for 8 segments of AT-MD simulations as system-dependent metrics. The distribution of protein size is measured by computing radius of gyration (Rg), its surface property by computing percent of hydrophobic accessible surface area (\%Hydrophobic ASA), and its global structure deviation from the ITASSER-generated model is measured by computing dRMS. As each segment started with different structure and seed number, the start points of each segment is unique. Segments 2 to 4 , $4 \mathrm{a}--4 \mathrm{c}$, and 5 experience similar general system and protein properties. 

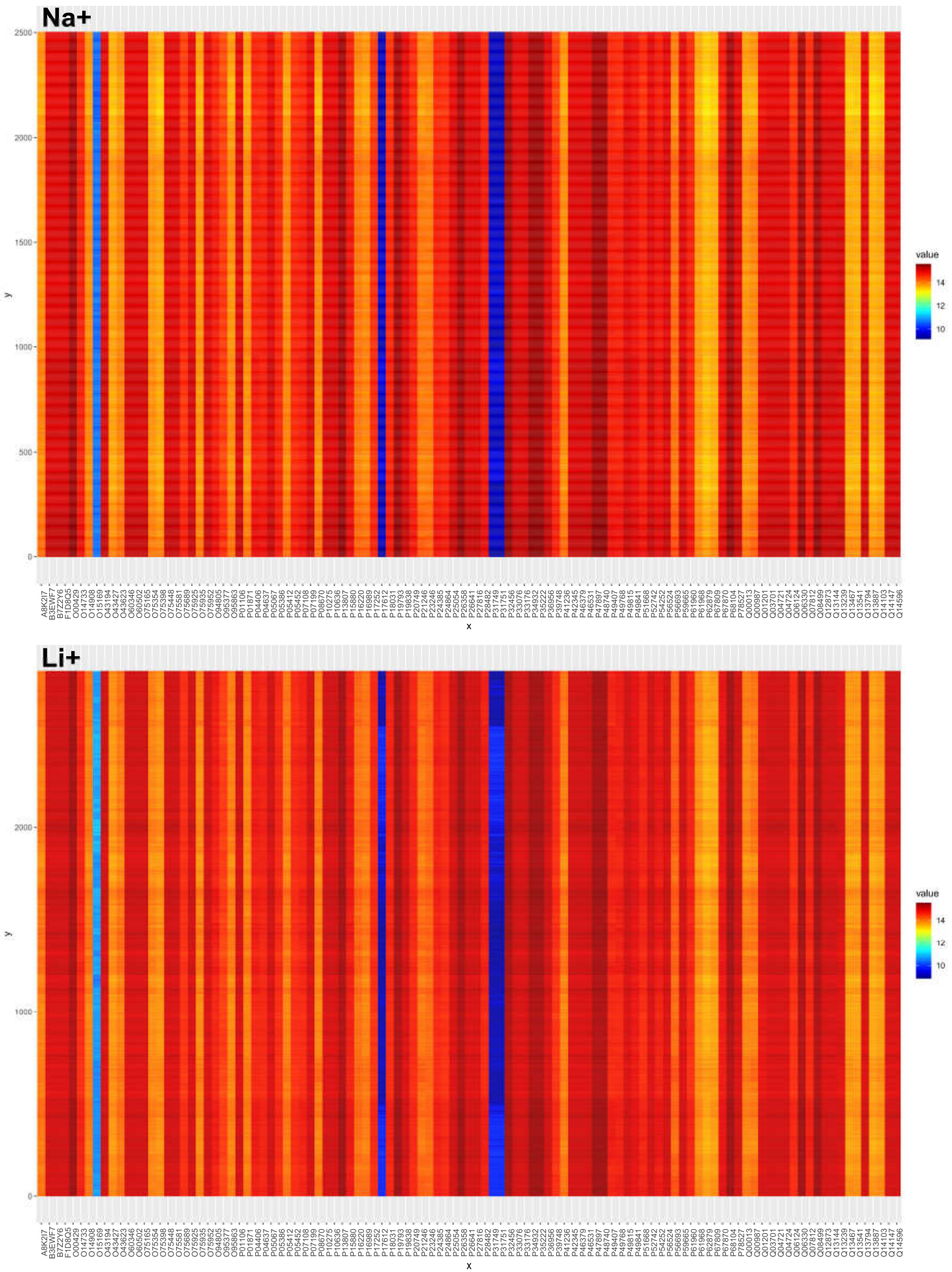

Figure S9. The trajectories of affinity values for binding patches 1 to 100 . 

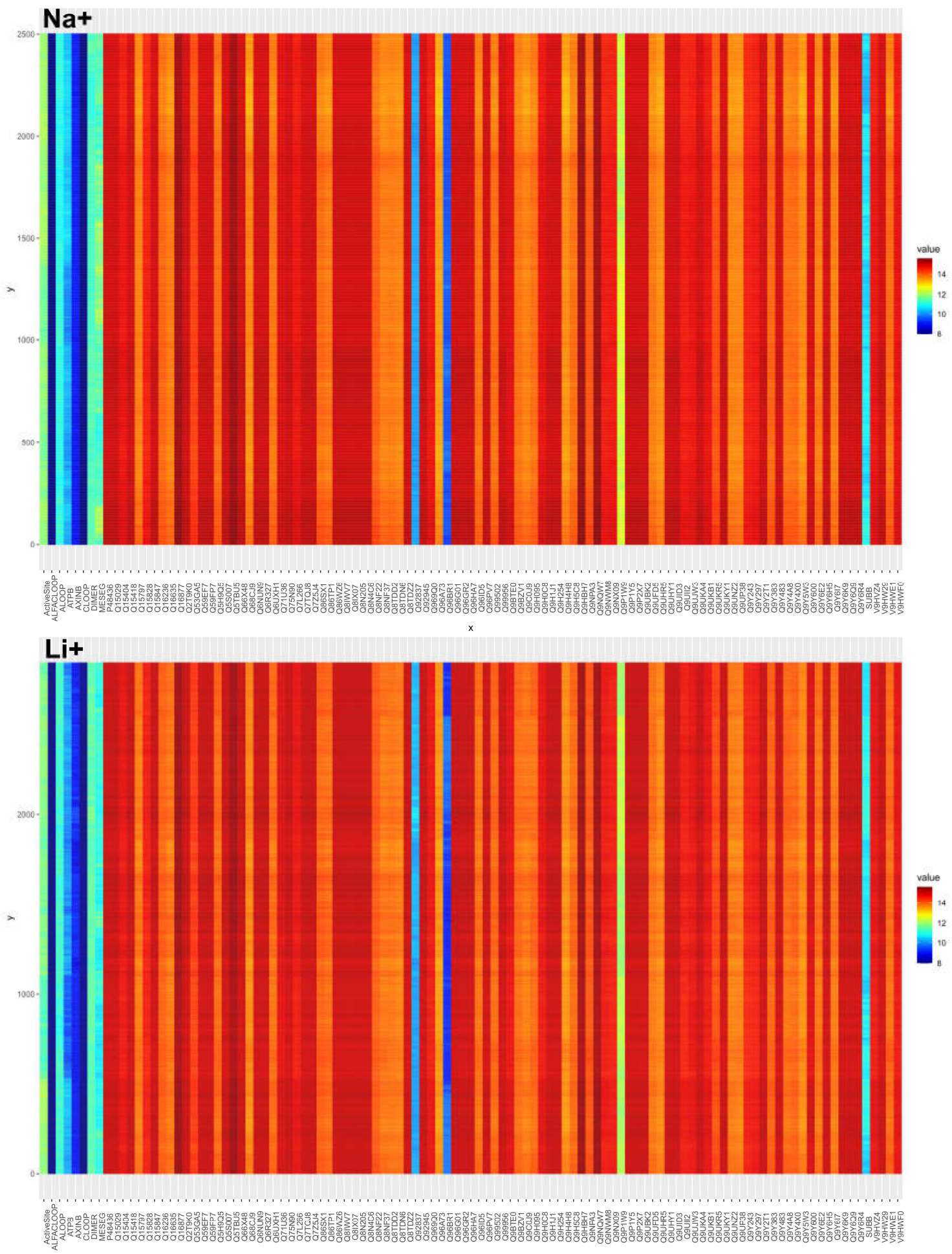

Figure S10. The trajectories of affinity values for binding patches 100 to end. 
Figures S9, S10. The trajectories of affinity values.

For every selected frame of AT-MD simulations, we computed the affinity of the considered GSK-3 $\beta$ binding patches ( $\mathrm{x}$-axis; uniprot ID) along the simulation time (y-axis). The simulation time is compressed to depict the changes in affinity values. The amount of the predicted affinity is presented by a color range. Darker red denotes a higher affinity of the binding patch for the partner. 


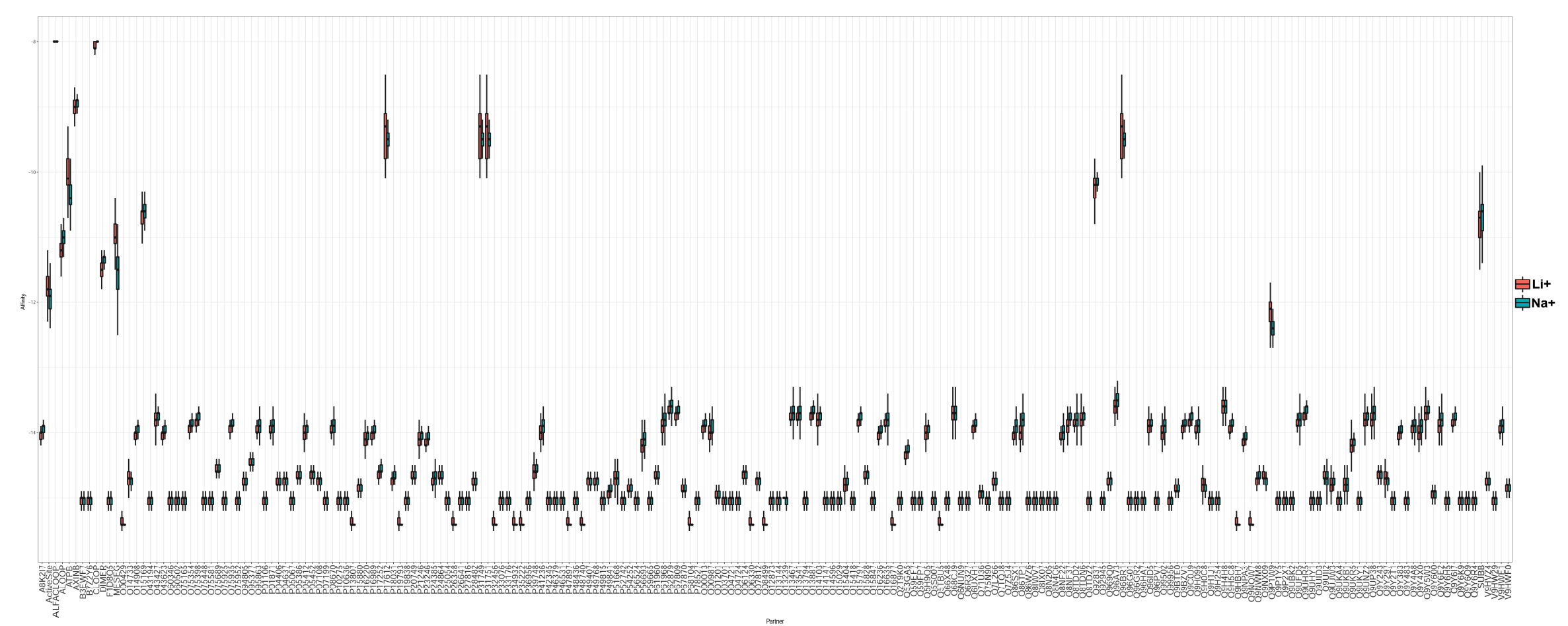

Figure S11. The effect of $\mathrm{LiCl}$ on the affinity of GSK-3 $\beta$ to its partners.

SEPAS-computed affinities of the considered binding sites on GSK-3 $\beta$ to the partners are presented as a bar-plot. For each binding site, the distribution of computed affinities is presented as a bar plot. The affinity values (in $\mathrm{kcal} / \mathrm{mol}$ ) are predicted on the basis of AT-MDgenerated ensembles for GSK-3 $\beta$ structure in the presence of $\mathrm{LiCl}$ (red bar) or $\mathrm{NaCl}$ (blue bar). 
Movies S1a, S1b. Dynamics of GSK-3 $\beta$ in the presence of LiCl.

The EDA-defined trajectory of GSK-3 $\beta$ global motion in the presence of $\mathrm{LiCl}$ is converted to a movie. N-terminal is defined by yellow and active site residues by blue surface. C-terminal tail is colored as light blue-white.

Movies S2a, S2b. Dynamics of GSK-3 $\beta$ in the presence of $\mathrm{NaCl}$.

The EDA-defined trajectory of GSK-3 $\beta$ global motion in the presence of $\mathrm{NaCl}$ is depicted as a movie. N-terminal is defined by yellow and active site residues by blue surface. $\mathrm{C}$-terminal tail is colored as light blue-white. 
Table S1. Comparison of GSK-3 $\beta$ secondary structures in the presence of sodium and lithium ions.

$\begin{array}{cccccccc} & & \text { alpha-Helix } & \text { beta-Bridge } & \text { Strand } & \mathbf{3 / 1 0} \text {-Helix } & \text { Turn } & \text { Bend } \\ \mathrm{Na} & \text { Average } & 0.3616 & 0.0264 & 0.2128 & 0.0434 & 0.1523 & 0.2033 \\ & \text { SEM } & 0.0002 & 0.0001 & 0.0001 & 0.0002 & 0.0002 & 0.0002 \\ \mathrm{~L} & \text { Average } & 0.3626 & 0.0264 & 0.2195 & 0.0342 & 0.1372 & 0.2208 \\ & \text { SEM } & 0.0001 & 0.0001 & 0.0001 & 0.0001 & 0.0002 & 0.0002 \\ & \text { p-value } & 3.545 \mathrm{E}-06 & 7.887 \mathrm{E}-01 & 0.000 \mathrm{E}+00 & 0.000 \mathrm{E}+00 & 0.000 \mathrm{E}+00 & 0.000 \mathrm{E}+00\end{array}$


Table S2. Comparison of GSK-3 $\beta$ affinities towards its partners in the presence of sodium and lithium ions in the three sets of AT-MD data.

\begin{tabular}{|c|c|c|c|c|c|c|}
\hline UI & MT & Gene names & $\begin{array}{l}z \text { score } \\
\text { set } 1\end{array}$ & $\begin{array}{l}z \text { score } \\
\text { set } 2\end{array}$ & $\begin{array}{l}z \text { score } \\
\text { set } 3\end{array}$ & Pathway \\
\hline P23246 & ECLAIR & SFPQ PSF & -74.6894 & -74.6605 & -74.5979 & \\
\hline Q86TP1 & ECLAIR & PRUNE1 PRUNE & -68.0919 & -67.6898 & -68.0328 & \\
\hline O75398 & ECLAIR & DEAF1 SPN ZMYND5 & -65.2013 & -64.3801 & -65.1735 & \\
\hline Q9C0J9 & ECLAIR & $\begin{array}{l}\text { BHLHE41 BHLHB3 } \\
\text { DEC2 SHARP1 }\end{array}$ & -60.3554 & -60.2498 & -60.4002 & \\
\hline Q86SX1 & ECLAIR & & -56.8936 & -56.5728 & -56.8682 & \\
\hline Q9H5C8 & ECLAIR & & -56.7044 & -55.8253 & -56.7689 & \\
\hline Q9Y6I7 & ECLAIR & WSB1 SWIP1 & -54.1544 & -53.6573 & -53.5191 & \\
\hline 075354 & ECLAIR & $\begin{array}{l}\text { ENTPD6 CD39L2 } \\
\text { IL6ST2 }\end{array}$ & -53.566 & -52.1569 & -52.8298 & \\
\hline Q13541 & ECLAIR & EIF4EBP1 & -51.589 & -50.896 & -50.4558 & CCKR, p53 glucose, p38 \\
\hline Q9UHR5 & ECLAIR & $\begin{array}{l}\text { SAP30BP HCNGP } \\
\text { HTRG HTRP }\end{array}$ & -50.6053 & -50.1716 & -50.7163 & \\
\hline Q9Y6E2 & ECLAIR & $\begin{array}{l}\text { BZW2 HSPC028 } \\
\text { MSTP017 }\end{array}$ & -50.896 & -50.149 & -50.7083 & \\
\hline Q15797 & ECLAIR & $\begin{array}{l}\text { SMAD1 BSP1 MADH1 } \\
\text { MADR1 }\end{array}$ & -49.7365 & -49.5329 & -49.4359 & Wnt, GRHR, TGF-beta \\
\hline Q16635 & ECLAIR & TAZ EFE2 G4.5 & -48.8977 & -49.1207 & -49.163 & \\
\hline Q14103 & ECLAIR & HNRNPD AUF1 HNRPD & -48.7994 & -49.093 & -49.159 & \\
\hline Q9UNZ2 & ECLAIR & NSFL1C UBXN2C & -48.8263 & -48.8739 & -49.1902 & \\
\hline Q16236 & ECLAIR & NFE2L2 NRF2 & -49.4756 & -48.5487 & -49.7312 & \\
\hline Q9UFD5 & ECLAIR & DKFZp434N181 & -48.2229 & -48.4791 & -48.3304 & \\
\hline Q9UP38 & ECLAIR & FZD1 & -48.8959 & -48.1996 & -48.4281 & $\begin{array}{l}\text { Wnt, Angiogenesis, Alz presenilin, } \\
\text { Cadherin }\end{array}$ \\
\hline Q8NF37 & ECLAIR & LPCAT1 AYTL2 PFAAP3 & -47.5128 & -47.228 & -47.9643 & \\
\hline Q6UXH1 & ECLAIR & $\begin{array}{l}\text { CRELD2 } \\
\text { UNQ185/PRO211 }\end{array}$ & -47.486 & -46.7162 & -46.7585 & \\
\hline O43623 & ECLAIR & SNAI2 SLUG SLUGH & -47.0675 & -46.4182 & -47.0308 & \\
\hline Q9BZV1 & ECLAIR & $\begin{array}{l}\text { UBXN6 UBXD1 } \\
\text { UBXDC2 }\end{array}$ & -46.4493 & -45.7058 & -46.1995 & \\
\hline P41236 & ECLAIR & PPP1R2 IPP2 & -46.107 & -45.7054 & -46.1574 & \\
\hline P16220 & ECLAIR & CREB1 & -45.7357 & -45.5393 & -45.9613 & $\begin{array}{l}\text { GRHR, CCKR, Apoptosis, } \\
\text { Enkephalin, Gi Gs, p38, bZIP }\end{array}$ \\
\hline O95863 & ECLAIR & SNAI1 SNAH & -45.9142 & -45.4823 & -45.6368 & CCKR \\
\hline Q9H095 & ECLAIR & IQCG DRC9 & -45.3863 & -45.168 & -45.2698 & \\
\hline P61968 & ECLAIR & LMO4 & -45.0883 & -44.5732 & -45.0096 & \\
\hline A8K2I7 & ECLAIR & & -45.137 & -44.2143 & -45.2031 & \\
\hline O14908 & ECLAIR & $\begin{array}{l}\text { GIPC1 C19orf3 GIPC } \\
\text { RGS19IP1 }\end{array}$ & -45.137 & -44.2143 & -45.2031 & \\
\hline Q9UKR5 & ECLAIR & $\begin{array}{l}\text { ERG28 C14orf1 AD-011 } \\
\text { HSPC288 x0006 }\end{array}$ & -43.7324 & -44.1274 & -44.2216 & \\
\hline
\end{tabular}




\begin{tabular}{|c|c|c|c|c|c|c|}
\hline Q92837 & PDB & FRAT1 & -45.3413 & -44.11 & -43.458 & \\
\hline Q99502 & ECLAIR & EYA1 & -44.8706 & -43.904 & -43.9798 & \\
\hline Q96A73 & ECLAIR & KIAA1191 P33MONOX & -44.2074 & -43.8933 & -44.4639 & \\
\hline Q9Y4X0 & ECLAIR & AMMECR1 & -43.8068 & -43.5779 & -44.0387 & \\
\hline Q00013 & ECLAIR & MPP1 DXS552E EMP55 & -43.5999 & -43.359 & -44.1142 & \\
\hline Q9Y383 & ECLAIR & LUC7L2 CGI-59 CGI-74 & -44.0576 & -43.0878 & -44.2576 & \\
\hline Q13887 & ECLAIR & KLF5 BTEB2 CKLF IKLF & -43.0291 & -42.9975 & -42.758 & \\
\hline P05412 & ECLAIR & JUN & -43.622 & -42.5624 & -43.1547 & $\begin{array}{l}\text { Angiogenesis, GRHR, CCKR, } \\
\text { Apoptosis, Inflammation, PDGF, } \\
\text { Huntington, B cell, FAS, Oxidative, } \\
\text { Ras, T cell, TGF-beta, Toll receptor }\end{array}$ \\
\hline 075935 & ECLAIR & DCTN3 DCTN22 & -42.1621 & -41.8408 & -42.334 & \\
\hline Q8TDN6 & ECLAIR & BRIX1 BRIX BXDC2 & -42.0426 & -41.1629 & -41.8326 & \\
\hline Q53GA5 & ECLAIR & & -41.3429 & -41.015 & -40.9506 & \\
\hline Q8NF22 & ECLAIR & FLJ00380 & -40.6248 & -40.5268 & -40.1475 & \\
\hline O43427 & ECLAIR & FIBP & -40.2382 & -40.3605 & -40.7684 & \\
\hline P62879 & ECLAIR & GNB2 & -40.3066 & -39.5342 & -39.8172 & $\begin{array}{l}\text { Wnt, GRHR, 5HT1, 5HT2, 5HT4, } \\
\text { Angiotensin, Adrenergic beta1, } \\
\text { Adrenergic beta2, Adrenergic beta3, } \\
\text { CRFR, Dopamine receptor, } \\
\text { Cannabinoid, Enkephalin, GABA } \\
\text { receptor, Gi Gs, Gq Go, } \\
\text { phototransduction, H1R, H2R, } \\
\text { Metabotropic II, Metabotropic III, } \\
\text { Muscarinic } 13 \text {, Muscarinic } 24 \text {, } \\
\text { Prodynorphin, Proenkephalin, } \\
\text { Proopiomelanocortin, Oxytocin } \\
\text { receptor, PI3K, TRHR }\end{array}$ \\
\hline Q5H9Q5 & ECLAIR & DKFZp781N0293 & -39.47 & -38.8495 & -39.2435 & \\
\hline P18031 & ECLAIR & PTPN1 PTP1B & -38.3174 & -38.8187 & -38.3209 & Cadherin \\
\hline P16989 & ECLAIR & YBX3 CSDA DBPA & -39.2156 & -38.6882 & -39.4453 & GRHR \\
\hline Q9NPA3 & ECLAIR & MID1IP1 MIG12 & -38.2394 & -37.9364 & -38.2879 & \\
\hline Q8TDD2 & ECLAIR & SP7 OSX & -38.0495 & -37.627 & -37.7799 & \\
\hline P56693 & ECLAIR & SoX10 & -36.1639 & -36.6482 & -36.7077 & \\
\hline P01871 & ECLAIR & IGHM & -36.5751 & -36.4137 & -36.8684 & B cell \\
\hline Q96ID5 & ECLAIR & IGSF21 & -35.1509 & -35.3293 & -35.2354 & \\
\hline Q13467 & ECLAIR & FZD5 C2orf31 & -35.1454 & -34.3585 & -34.9236 & $\begin{array}{l}\text { Wnt, Angiogenesis, Alz presenilin, } \\
\text { Cadherin }\end{array}$ \\
\hline Q9Y4A8 & ECLAIR & NFE2L3 NRF3 & -33.6829 & -33.5181 & -34.2371 & \\
\hline
\end{tabular}




\begin{tabular}{|c|c|c|c|c|c|c|}
\hline $\mathrm{P} 17252$ & ECLAIR & PRKCA PKCA PRKACA & -33.1752 & -33.4108 & -32.1835 & $\begin{array}{l}\text { Wnt, Angiogenesis, GRHR, CCKR, } \\
\text { Apoptosis, PDGF, Alz secretase, } \\
5 H T 2, \text { Adrenergic alpha, } \\
\text { Angiotensin, EGFR, Endothelin, } \\
\text { FGF, Gq Go, H1R, Muscarinic } 13 \text {, } \\
\text { Oxytocin receptor, TRHR, VEGF }\end{array}$ \\
\hline P08670 & ECLAIR & VIM & -33.6259 & -32.985 & -33.276 & \\
\hline V9HWE1 & ECLAIR & HEL113 VIM & -33.6259 & -32.985 & -33.276 & \\
\hline Q9NWM8 & ECLAIR & $\begin{array}{l}\text { FKBP14 FKBP22 } \\
\text { UNQ322/PRO381 }\end{array}$ & -32.8603 & -32.151 & -31.7845 & \\
\hline P24385 & ECLAIR & CCND1 BCL1 PRAD1 & -31.9939 & -31.9442 & -31.7194 & Wnt, CCKR, Cell cycle, PI3K \\
\hline P67809 & ECLAIR & YBX1 NSEP1 YB1 & -31.391 & -31.4092 & -31.3639 & \\
\hline Q00987 & ECLAIR & MDM2 & -29.7549 & -29.7421 & -29.6187 & $\begin{array}{l}\text { Insulin PKB, p53 loops1, p53 loops2, } \\
\text { p53, Ubiquitin }\end{array}$ \\
\hline O15169 & PDB & AXIN1 AXIN & -30.0559 & -29.5944 & -29.0399 & Wnt, Angiogenesis \\
\hline P39748 & ECLAIR & FEN1 RAD2 & -29.8141 & -29.4299 & -29.0903 & \\
\hline Q9Y5W3 & ECLAIR & KLF2 LKLF & -24.3341 & -24.2041 & -23.9412 & \\
\hline B3EWF7 & ECLAIR & EPM2A & -23.9085 & -23.8008 & -22.5313 & \\
\hline B7Z2Y6 & ECLAIR & & -23.9085 & -23.8008 & -22.5313 & \\
\hline F1D8Q5 & ECLAIR & $\begin{array}{l}\text { NR2B1 RXRA } \\
\text { hCG_18150 }\end{array}$ & -23.9085 & -23.8008 & -22.5313 & \\
\hline O43194 & ECLAIR & GPR39 & -23.9085 & -23.8008 & -22.5313 & \\
\hline O60346 & ECLAIR & $\begin{array}{l}\text { PHLPP1 KIAA0606 } \\
\text { PHLPP PLEKHE1 SCOP }\end{array}$ & -23.9085 & -23.8008 & -22.5313 & \\
\hline 060502 & ECLAIR & $\begin{array}{l}\text { OGA HEXC KIAA0679 } \\
\text { MEA5 MGEA5 }\end{array}$ & -23.9085 & -23.8008 & -22.5313 & \\
\hline O75165 & ECLAIR & $\begin{array}{l}\text { DNAJC13 KIAA0678 } \\
\text { RME8 }\end{array}$ & -23.9085 & -23.8008 & -22.5313 & \\
\hline O75448 & ECLAIR & $\begin{array}{l}\text { MED24 ARC100 CRSP4 } \\
\text { DRIP100 KIAA0130 } \\
\text { THRAP4 TRAP100 }\end{array}$ & -23.9085 & -23.8008 & -22.5313 & \\
\hline O75581 & ECLAIR & LRP6 & -23.9085 & -23.8008 & -22.5313 & Wnt, Alz presenilin \\
\hline O75925 & ECLAIR & PIAS1 DDXBP1 & -23.9085 & -23.8008 & -22.5313 & Interferon, JAK/STAT \\
\hline O75952 & ECLAIR & CABYR CBP86 FSP2 & -23.9085 & -23.8008 & -22.5313 & \\
\hline P01106 & ECLAIR & MYC BHLHE39 & -23.9085 & -23.8008 & -22.5313 & $\begin{array}{l}\text { Wnt, CCKR, PDGF, Interleukin, } \\
\text { Oxidative, p53 loops } 2\end{array}$ \\
\hline P05067 & ECLAIR & APP A4 AD1 & -23.9085 & -23.8008 & -22.5313 & $\begin{array}{l}\text { Alz presenilin, Alz secretase, } \\
\text { Coagulation }\end{array}$ \\
\hline P07199 & ECLAIR & CENPB & -23.9085 & -23.8008 & -22.5313 & \\
\hline P10275 & ECLAIR & AR DHTR NR3C4 & -23.9085 & -23.8008 & -22.5313 & GRHR \\
\hline P10636 & ECLAIR & $\begin{array}{l}\text { MAPT MAPTL MTBT1 } \\
\text { TAU }\end{array}$ & -23.9085 & -23.8008 & -22.5313 & \\
\hline
\end{tabular}




\begin{tabular}{|c|c|c|c|c|c|c|}
\hline P19838 & ECLAIR & NFKB1 & -23.9085 & -23.8008 & -22.5313 & $\begin{array}{l}\text { Apoptosis, Inflammation, B cell, T } \\
\text { cell, Toll receptor }\end{array}$ \\
\hline P25054 & ECLAIR & APC DP2.5 & -23.9085 & -23.8008 & -22.5313 & Wnt, Angiogenesis \\
\hline P26641 & ECLAIR & EEF1G EF1G PRO1608 & -23.9085 & -23.8008 & -22.5313 & \\
\hline P27816 & ECLAIR & MAP4 & -23.9085 & -23.8008 & -22.5313 & \\
\hline P33076 & ECLAIR & CIITA MHC2TA & -23.9085 & -23.8008 & -22.5313 & \\
\hline P33176 & ECLAIR & KIF5B KNS KNS1 & -23.9085 & -23.8008 & -22.5313 & \\
\hline P36956 & ECLAIR & $\begin{array}{l}\text { SREBF1 BHLHD1 } \\
\text { SREBP1 }\end{array}$ & -23.9085 & -23.8008 & -22.5313 & \\
\hline P42345 & ECLAIR & $\begin{array}{l}\text { MTOR FRAP FRAP1 } \\
\text { FRAP2 RAFT1 RAPT1 }\end{array}$ & -23.9085 & -23.8008 & -22.5313 & $\begin{array}{l}\text { PDGF, Hypoxia, Interleukin, p53 } \\
\text { glucose }\end{array}$ \\
\hline P46379 & ECLAIR & BAG6 BAT3 G3 & -23.9085 & -23.8008 & -22.5313 & \\
\hline P46531 & ECLAIR & NOTCH1 TAN1 & -23.9085 & -23.8008 & -22.5313 & Angiogenesis, Alz presenilin, Notch \\
\hline P48436 & ECLAIR & Sox9 & -23.9085 & -23.8008 & -22.5313 & \\
\hline P49815 & ECLAIR & TSC2 TSC4 & -23.9085 & -23.8008 & -22.5313 & Insulin PKB, p53 glucose \\
\hline P52742 & ECLAIR & $\begin{array}{l}\text { ZNF135 ZNF61 } \\
\text { ZNF78L1 }\end{array}$ & -23.9085 & -23.8008 & -22.5313 & \\
\hline P56524 & ECLAIR & HDAC4 KIAA0288 & -23.9085 & -23.8008 & -22.5313 & \\
\hline P59665 & ECLAIR & $\begin{array}{l}\text { DEFA1 DEF1 DEFA2 } \\
\text { MRS; DEFA1B }\end{array}$ & -23.9085 & -23.8008 & -22.5313 & \\
\hline P78527 & ECLAIR & PRKDC HYRC HYRC1 & -23.9085 & -23.8008 & -22.5313 & \\
\hline Q03701 & ECLAIR & CEBPZ CBF2 & -23.9085 & -23.8008 & -22.5313 & \\
\hline Q04721 & ECLAIR & NOTCH2 & -23.9085 & -23.8008 & -22.5313 & Angiogenesis, Alz presenilin, Notch \\
\hline Q04724 & ECLAIR & TLE1 & -23.9085 & -23.8008 & -22.5313 & Wnt \\
\hline Q12873 & ECLAIR & CHD3 & -23.9085 & -23.8008 & -22.5313 & \\
\hline Q13144 & ECLAIR & EIF2B5 EIF2BE & -23.9085 & -23.8008 & -22.5313 & \\
\hline Q13794 & ECLAIR & PMAIP1 NOXA & -23.9085 & -23.8008 & -22.5313 & p53 \\
\hline Q14147 & ECLAIR & $\begin{array}{l}\text { DHX34 DDX34 } \\
\text { KIAA0134 }\end{array}$ & -23.9085 & -23.8008 & -22.5313 & \\
\hline Q14596 & ECLAIR & $\begin{array}{l}\text { NBR1 1A13B KIAA0049 } \\
\text { M17S2 MIG19 }\end{array}$ & -23.9085 & -23.8008 & -22.5313 & \\
\hline Q15029 & ECLAIR & $\begin{array}{l}\text { EFTUD2 KIAA0031 } \\
\text { SNRP116 }\end{array}$ & -23.9085 & -23.8008 & -22.5313 & \\
\hline Q15418 & ECLAIR & $\begin{array}{l}\text { RPS6KA1 MAPKAPK1A } \\
\text { RSK1 }\end{array}$ & -23.9085 & -23.8008 & -22.5313 & $\begin{array}{l}\text { CCKR, PDGF, Insulin MAPK, } \\
\text { Interleukin, Ras }\end{array}$ \\
\hline Q15847 & ECLAIR & $\begin{array}{l}\text { ADIRF AFRO APM2 } \\
\text { C10orf116 }\end{array}$ & -23.9085 & -23.8008 & -22.5313 & \\
\hline Q2T9K0 & ECLAIR & TMEM44 & -23.9085 & -23.8008 & -22.5313 & \\
\hline Q59EF7 & ECLAIR & & -23.9085 & -23.8008 & -22.5313 & \\
\hline Q59FP7 & ECLAIR & & -23.9085 & -23.8008 & -22.5313 & \\
\hline Q5S007 & ECLAIR & LRRK2 PARK8 & -23.9085 & -23.8008 & -22.5313 & \\
\hline Q66X48 & ECLAIR & MHC2TA & -23.9085 & -23.8008 & -22.5313 & \\
\hline Q6NUN9 & ECLAIR & ZNF746 PARIS & -23.9085 & -23.8008 & -22.5313 & \\
\hline
\end{tabular}




\begin{tabular}{|c|c|c|c|c|c|c|}
\hline Q6R327 & ECLAIR & RICTOR KIAA1999 & -23.9085 & -23.8008 & -22.5313 & \\
\hline Q75N90 & ECLAIR & FBN3 KIAA1776 & -23.9085 & -23.8008 & -22.5313 & \\
\hline Q7TQJ8 & ECLAIR & Wtip & -23.9085 & -23.8008 & -22.5313 & \\
\hline Q7Z5J4 & ECLAIR & RAI1 KIAA1820 & -23.9085 & -23.8008 & -22.5313 & \\
\hline Q86WZ6 & ECLAIR & ZNF227 & -23.9085 & -23.8008 & -22.5313 & \\
\hline Q8IWV7 & ECLAIR & UBR1 & -23.9085 & -23.8008 & -22.5313 & \\
\hline Q8IX07 & ECLAIR & ZFPM1 FOG1 ZFN89A & -23.9085 & -23.8008 & -22.5313 & \\
\hline Q8N205 & ECLAIR & SYNE4 C19orf46 & -23.9085 & -23.8008 & -22.5313 & \\
\hline Q8N4C6 & ECLAIR & NIN KIAA1565 & -23.9085 & -23.8008 & -22.5313 & PDGF \\
\hline Q8TDZ2 & ECLAIR & MICAL1 MICAL NICAL & -23.9085 & -23.8008 & -22.5313 & \\
\hline Q92945 & ECLAIR & KHSRP FUBP2 & -23.9085 & -23.8008 & -22.5313 & \\
\hline Q96G01 & ECLAIR & BICD1 & -23.9085 & -23.8008 & -22.5313 & \\
\hline Q96GR2 & ECLAIR & $\begin{array}{l}\text { ACSBG1 BGM } \\
\text { KIAA0631 LPD }\end{array}$ & -23.9085 & -23.8008 & -22.5313 & \\
\hline Q96HA7 & ECLAIR & TONSL IKBR NFKBIL2 & -23.9085 & -23.8008 & -22.5313 & \\
\hline Q96PV7 & ECLAIR & $\begin{array}{l}\text { FAM193B IRIZIO } \\
\text { KIAA1931 }\end{array}$ & -23.9085 & -23.8008 & -22.5313 & \\
\hline Q99956 & ECLAIR & DUSP9 MKP4 & -23.9085 & -23.8008 & -22.5313 & Oxidative \\
\hline Q9H1J1 & ECLAIR & UPF3A RENT3A UPF3 & -23.9085 & -23.8008 & -22.5313 & \\
\hline Q9H254 & ECLAIR & $\begin{array}{l}\text { SPTBN4 KIAA1642 } \\
\text { SPTBN3 }\end{array}$ & -23.9085 & -23.8008 & -22.5313 & Integrin \\
\hline Q9P1Y5 & ECLAIR & CAMSAP3 KIAA1543 & -23.9085 & -23.8008 & -22.5313 & \\
\hline Q9P2X7 & ECLAIR & DELEC1 CTS9 DEC1 & -23.9085 & -23.8008 & -22.5313 & \\
\hline Q9UBK2 & ECLAIR & $\begin{array}{l}\text { PPARGC1A LEM6 } \\
\text { PGC1 PGC1A } \\
\text { PPARGC1 }\end{array}$ & -23.9085 & -23.8008 & -22.5313 & \\
\hline Q9UHY1 & ECLAIR & NRBP1 BCON3 NRBP & -23.9085 & -23.8008 & -22.5313 & \\
\hline Q9UID3 & ECLAIR & $\begin{array}{l}\text { VPS51 ANG2 C11orf2 } \\
\text { C11orf3 FFR PP5382 }\end{array}$ & -23.9085 & -23.8008 & -22.5313 & \\
\hline Q9UKA4 & ECLAIR & $\begin{array}{l}\text { AKAP11 AKAP220 } \\
\text { KIAA0629 }\end{array}$ & -23.9085 & -23.8008 & -22.5313 & \\
\hline Q9UKY1 & ECLAIR & $\mathrm{ZHX1}$ & -23.9085 & -23.8008 & -22.5313 & \\
\hline Q9Y2T1 & ECLAIR & AXIN2 & -23.9085 & -23.8008 & -22.5313 & Wnt, Angiogenesis \\
\hline Q9Y483 & ECLAIR & MTF2 PCL2 & -23.9085 & -23.8008 & -22.5313 & \\
\hline Q9Y6H5 & ECLAIR & SNCAIP & -23.9085 & -23.8008 & -22.5313 & Parkinson \\
\hline Q9Y6K9 & ECLAIR & IKBKG FIP3 NEMO & -23.9085 & -23.8008 & -22.5313 & \\
\hline Q9Y6Q9 & ECLAIR & $\begin{array}{l}\text { NCOA3 AIB1 BHLHE42 } \\
\text { RAC3 TRAM1 }\end{array}$ & -23.9085 & -23.8008 & -22.5313 & GRHR \\
\hline Q9Y6R4 & ECLAIR & $\begin{array}{l}\text { MAP3K4 KIAA0213 } \\
\text { MAPKKK4 MEKK4 } \\
\text { MTK1 }\end{array}$ & -23.9085 & -23.8008 & -22.5313 & $\begin{array}{l}\text { GRHR, Inflammation, PDGF, EGFR, } \\
\text { FGF, Integrin, Interleukin, Oxidative, } \\
\text { p38, Ras }\end{array}$ \\
\hline V9HW29 & ECLAIR & HEL-S-61 & -23.9085 & -23.8008 & -22.5313 & \\
\hline Q9UJW3 & ECLAIR & DNMT3L & -22.9392 & -22.8827 & -22.7528 & \\
\hline P21246 & ECLAIR & PTN HBNF1 NEGF1 & -22.4672 & -21.9429 & -21.9149 & \\
\hline Q15404 & ECLAIR & RSU1 RSP1 & -21.6598 & -20.8422 & -20.8464 & \\
\hline
\end{tabular}




\begin{tabular}{|c|c|c|c|c|c|c|}
\hline P49768 & ECLAIR & PSEN1 AD3 PS1 PSNL1 & -21.1403 & -20.4927 & -21.1265 & Alz presenilin, Alz secretase, Notch \\
\hline Q15828 & ECLAIR & CST6 & -21.2034 & -20.1117 & -20.1647 & \\
\hline Q68CJ9 & ECLAIR & $\begin{array}{l}\text { CREB3L3 CREBH } \\
\text { HYST1481 }\end{array}$ & -20.0958 & -19.6923 & -19.9989 & Gi Gs, bZIP \\
\hline P67870 & ECLAIR & CSNK2B CK2N G5A & -17.9545 & -16.9326 & -16.9066 & Wnt, Parkinson, Cadherin \\
\hline P04406 & ECLAIR & $\begin{array}{l}\text { GAPDH GAPD } \\
\text { CDABP0047 OK/SW- } \\
\text { cl.12 }\end{array}$ & -16.7563 & -16.5094 & -16.0619 & Huntington, Glycolysis \\
\hline V9HVZ4 & ECLAIR & HEL-S-162eP & -16.7563 & -16.5094 & -16.0619 & \\
\hline Q9H4H8 & ECLAIR & FAM83D C20orf129 & -15.6619 & -15.3549 & -15.3352 & \\
\hline P49841 & PDB & GSK3B & -15.264 & -14.7482 & -14.2012 & $\begin{array}{l}\text { Wnt, Angiogenesis, GRHR, CCKR, } \\
\text { Alz presenilin, PDGF, Cadherin, } \\
\text { Hedgehog, Gi Gs, Insulin PKB, } \\
\text { Interleukin, PI3K, Ras }\end{array}$ \\
\hline Q07812 & ECLAIR & BAX BCL2L4 & -13.4329 & -13.3228 & -13.0873 & CCKR, Apoptosis, Huntington, p53 \\
\hline O75689 & ECLAIR & ADAP1 CENTA1 & -13.5222 & -13.252 & -13.0834 & \\
\hline Q06124 & ECLAIR & $\begin{array}{l}\text { PTPN11 PTP2C } \\
\text { SHPTP2 }\end{array}$ & -11.2917 & -10.5201 & -10.7706 & $\begin{array}{l}\text { Angiogenesis, CCKR, FGF, } \\
\text { Interferon }\end{array}$ \\
\hline P54252 & ECLAIR & $\begin{array}{l}\text { ATXN3 ATX3 MJD MJD1 } \\
\text { SCA3 }\end{array}$ & -10.7856 & -10.5021 & -10.3807 & \\
\hline V9HWF0 & ECLAIR & HEL-S-28 & -10.3986 & -10.1674 & -10.3397 & \\
\hline Q7L266 & ECLAIR & ASRGL1 ALP CRASH & -5.60188 & -5.11786 & -4.89828 & \\
\hline P15880 & ECLAIR & RPS2 RPS4 & -2.5654 & -2.67878 & -3.15171 & \\
\hline P61960 & ECLAIR & UFM1 C13orf20 BM-002 & -2.8068 & -2.46456 & -2.3734 & \\
\hline O94805 & ECLAIR & ACTL6B ACTL6 BAF53B & -3.27743 & -1.86271 & -2.17812 & \\
\hline Q9Y243 & ECLAIR & AKT3 PKBG & -2.68885 & -1.76185 & -1.87696 & $\begin{array}{l}\text { Angiogenesis, Apoptosis, } \\
\text { Inflammation, Huntington, EGFR, } \\
\text { Endothelin, FGF, Hypoxia, } \\
\text { Interleukin, p53 loops2, p53, p53 } \\
\text { glucose, PI3K, Ras, T cell }\end{array}$ \\
\hline P20749 & ECLAIR & BCL3 BCL4 D19S37 & -1.59443 & -1.29013 & -0.97813 & Inflammation \\
\hline Q969Q0 & ECLAIR & RPL36AL & -1.93255 & -0.93685 & -0.74992 & \\
\hline Q01201 & ECLAIR & RELB & -0.5068 & -0.40031 & -0.04909 & Apoptosis, Inflammation \\
\hline P51668 & ECLAIR & $\begin{array}{l}\text { UBE2D1 SFT UBC5A } \\
\text { UBCH5 UBCH5A }\end{array}$ & -0.9578 & -0.23504 & 0.362794 & Ubiquitin \\
\hline P04637 & ECLAIR & TP53 P53 & 0.17628 & 0.688198 & 0.171787 & $\begin{array}{l}\text { Wnt, Apoptosis, Huntington, p53 } \\
\text { loops1, p53 loops2, p53, p53 } \\
\text { glucose }\end{array}$ \\
\hline P07108 & ECLAIR & $\mathrm{DBI}$ & 0.347937 & 0.768906 & 0.577441 & \\
\hline Q9Y600 & ECLAIR & CSAD CSD & 0.710882 & 2.202386 & 1.279282 & GABA \\
\hline
\end{tabular}




\begin{tabular}{|c|c|c|c|c|c|c|}
\hline Q9UKB1 & ECLAIR & $\begin{array}{l}\text { FBXW11 BTRCP2 } \\
\text { FBW1B FBXW1B } \\
\text { KIAA0696 }\end{array}$ & 3.26253 & 4.689852 & 4.77031 & Wnt, Parkinson, Hedgehog \\
\hline P28482 & ECLAIR & $\begin{array}{l}\text { MAPK1 ERK2 PRKM1 } \\
\text { PRKM2 }\end{array}$ & 4.836632 & 5.584961 & 5.253645 & $\begin{array}{l}\text { Angiogenesis, GRHR, CCKR, } \\
\text { Apoptosis, Inflammation, PDGF, Alz } \\
\text { secretase, Parkinson, Angiotensin, B } \\
\text { cell, EGFR, Endothelin, FGF, Insulin } \\
\text { MAPK, Integrin, Interferon, } \\
\text { Interleukin, Ras, T cell, TGF-beta, } \\
\text { Toll receptor, VEGF }\end{array}$ \\
\hline P05452 & ECLAIR & CLEC3B TNA & 6.097749 & 6.664645 & 6.414166 & \\
\hline Q71U36 & ECLAIR & TUBA1A TUBA3 & 8.538065 & 8.084316 & 8.14823 & GRHR \\
\hline O95377 & ECLAIR & GJB5 & 7.652942 & 8.089385 & 7.390061 & \\
\hline P49407 & ECLAIR & ARRB1 ARR1 & 9.275802 & 9.833343 & 9.712214 & $\begin{array}{l}\text { Wnt, Inflammation, Angiotensin, Gi } \\
\text { Gs }\end{array}$ \\
\hline P05386 & ECLAIR & RPLP1 RRP1 & 9.271918 & 10.11818 & 9.118061 & \\
\hline P24864 & ECLAIR & CCNE1 CCNE & 9.928235 & 10.73775 & 11.03883 & $\begin{array}{l}\text { Parkinson, Cell cycle, p53 loops2, } \\
\text { p53 }\end{array}$ \\
\hline Q9Y297 & ECLAIR & $\begin{array}{l}\text { BTRC BTRCP FBW1A } \\
\text { FBXW1A }\end{array}$ & 11.81133 & 12.52212 & 12.45255 & Wnt, Hedgehog \\
\hline Q9UII2 & ECLAIR & ATP5IF1 ATPI ATPIF1 & 13.77945 & 14.26679 & 14.59521 & \\
\hline 014733 & ECLAIR & $\begin{array}{l}\text { MAP2K7 JNKK2 MEK7 } \\
\text { MKK7 PRKMK7 SKK4 }\end{array}$ & 17.86303 & 17.50681 & 17.8771 & $\begin{array}{l}\text { GRHR, Apoptosis, Huntington, } \\
\text { EGFR, FGF, Ras }\end{array}$ \\
\hline Q9H0C8 & ECLAIR & ILKAP & 19.67905 & 20.39103 & 20.15738 & \\
\hline Q13239 & ECLAIR & SLA SLAP SLAP1 & 22.51561 & 22.86296 & 22.75332 & \\
\hline Q9NX09 & ECLAIR & DDIT4 REDD1 RTP801 & 22.97266 & 23.92128 & 22.81046 & \\
\hline Q9BTE0 & ECLAIR & NAT9 EBS & 32.86411 & 33.26317 & 33.10973 & \\
\hline 000429 & ECLAIR & DNM1L DLP1 DRP1 & 35.16111 & 35.9946 & 36.80207 & \\
\hline P13807 & ECLAIR & GYS1 GYS & 35.16111 & 35.9946 & 36.80207 & Gi Gs \\
\hline P19793 & ECLAIR & RXRA NR2B1 & 35.16111 & 35.9946 & 36.80207 & Vitamin D \\
\hline P26358 & ECLAIR & $\begin{array}{l}\text { DNMT1 AIM CXXC9 } \\
\text { DNMT }\end{array}$ & 35.16111 & 35.9946 & 36.80207 & \\
\hline P32456 & ECLAIR & GBP2 & 35.16111 & 35.9946 & 36.80207 & \\
\hline P34932 & ECLAIR & HSPA4 APG2 & 35.16111 & 35.9946 & 36.80207 & \\
\hline P35222 & ECLAIR & $\begin{array}{l}\text { CTNNB1 CTNNB } \\
\text { OK/SW-cl.35 PRO2286 }\end{array}$ & 35.16111 & 35.9946 & 36.80207 & $\begin{array}{l}\text { Wnt, Angiogenesis, GRHR, CCKR, } \\
\text { Alz presenilin, Cadherin, p53 loops } 2\end{array}$ \\
\hline P47897 & ECLAIR & QARS1 QARS & 35.16111 & 35.9946 & 36.80207 & Heme \\
\hline P48740 & ECLAIR & $\begin{array}{l}\text { MASP1 CRARF } \\
\text { CRARF1 PRSS5 }\end{array}$ & 35.16111 & 35.9946 & 36.80207 & \\
\hline P68104 & ECLAIR & $\begin{array}{l}\text { EEF1A1 EEF1A EF1A } \\
\text { LENG7 }\end{array}$ & 35.16111 & 35.9946 & 36.80207 & \\
\hline
\end{tabular}




\begin{tabular}{|c|c|c|c|c|c|c|}
\hline Q06330 & ECLAIR & $\begin{array}{l}\text { RBPJ IGKJRB IGKJRB1 } \\
\text { RBPJK RBPSUH }\end{array}$ & 35.16111 & 35.9946 & 36.80207 & Angiogenesis, Alz presenilin, Notch \\
\hline Q08499 & ECLAIR & PDE4D DPDE3 & 35.16111 & 35.9946 & 36.80207 & \\
\hline Q16877 & ECLAIR & PFKFB4 & 35.16111 & 35.9946 & 36.80207 & \\
\hline Q5TBU5 & ECLAIR & hCG_1773630 & 35.16111 & 35.9946 & 36.80207 & \\
\hline Q9HBH7 & ECLAIR & BEX1 & 35.16111 & 35.9946 & 36.80207 & \\
\hline Q9NQW7 & ECLAIR & $\begin{array}{l}\text { XPNPEP1 XPNPEPL } \\
\text { XPNPEPL1 }\end{array}$ & 35.16111 & 35.9946 & 36.80207 & \\
\hline P17612 & I3D & PRKACA PKACA & 44.46108 & 44.04368 & 43.84137 & $\begin{array}{l}\text { CCKR, Inflammation, } 5 \mathrm{HT} 1 \text {, } \\
\text { Adrenergic beta1, Adrenergic beta2, } \\
\text { Dopamine receptor, Endothelin, } \\
\text { Enkephalin, GABA receptor, Gi Gs, } \\
\text { phototransduction, H2R, } \\
\text { Metabotropic I, Metabotropic II, } \\
\text { Metabotropic III, Muscarinic } 24 \text {, } \\
\text { Nicotine }\end{array}$ \\
\hline P31749 & PDB & AKT1 PKB RAC & 44.46108 & 44.04368 & 43.84137 & $\begin{array}{l}\text { Angiogenesis, GRHR, CCKR, } \\
\text { Apoptosis, Inflammation, Huntington, } \\
\text { EGFR, Endothelin, FAS, FGF, } \\
\text { Hypoxia, Insulin PKB, Interleukin, } \\
\text { p53 loops2, p53, p53 glucose, PI3K, } \\
\text { Ras, T cell, VEGF }\end{array}$ \\
\hline P31751 & PDB & AKT2 & 44.46108 & 44.04368 & 43.84137 & $\begin{array}{l}\text { Angiogenesis, Apoptosis, } \\
\text { Inflammation, PDGF, Huntington, } \\
\text { EGFR, Endothelin, FAS, FGF, } \\
\text { Hypoxia, Insulin PKB, Interleukin, } \\
\text { p53 loops2, p53, p53 glucose, PI3K, } \\
\text { T cell }\end{array}$ \\
\hline Q96BR1 & I3D & SGK3 CISK SGKL & 44.46108 & 44.04368 & 43.84137 & \\
\hline Q9P1W9 & I3D & PIM2 & 104.5741 & 104.3173 & 104.5664 & \\
\hline
\end{tabular}

The UniProt ID (UI), the method by which the partner was determined by Interactome INSIDER (MT), Gene name, and the PANTHER-determined pathways related to each specific GSK-3 $\beta$ partner are depicted in separtae columns. The comparison between affinities in LiCl- and NaCl-containing environments ( $z$ scores) for three sets of AT-MD trajectory data are also included. Colors in $z$ score columns are indicative of changes in affinity. Blue and orange indicate a lower and a higher affinity in the presence of $\mathrm{LiCl}$ than in the presence of $\mathrm{NaCl}$, respectively. Gray indicates no significant difference between affinities in the two environments determined by two-sample $t$ test ( $z$ score); green indicates no significant affinity difference common in two-sample $t$ test and Tukey's test; and yellow means no significant affinity difference in the two environments shared in two-sample $t$ test, Tukey's test, and Dunnett's test. The pathway full names are summarized in Table S3. 
Table S3. Comparison of $z$ score values in different segments of MD simulations for set 1 data series.

\begin{tabular}{|c|c|c|c|c|c|c|c|c|c|c|}
\hline A8K217 & $\begin{array}{l}\text { Regular } \\
\text { MD } \\
7.88\end{array}$ & $\begin{array}{l}\text { AT-MD } \\
\text { Seg.1 } \\
10.88\end{array}$ & $\begin{array}{l}\text { AT-MD } \\
\text { Seg.2 } \\
1.09\end{array}$ & $\begin{array}{l}\text { AT-MD } \\
\text { Seg.3 } \\
-26.44\end{array}$ & $\begin{array}{l}\text { AT-MD } \\
\text { Seg.4 } \\
-56.17\end{array}$ & $\begin{array}{l}\text { AT-MD } \\
\text { Seg.4a } \\
-37.68\end{array}$ & $\begin{array}{l}\text { AT-MD } \\
\text { Seg.4b } \\
-27.40\end{array}$ & $\begin{array}{c}\text { AT-MD } \\
\text { Seg.4c } \\
-37.78\end{array}$ & $\begin{array}{l}\text { AT-MD } \\
\text { Seg.5 } \\
-25.79\end{array}$ & EDA \\
\hline B3EWF7 & 8.86 & 23.01 & 27.49 & -11.58 & -38.36 & -24.11 & -18.97 & -45.66 & -13.29 & -86.32 \\
\hline B7Z2Y6 & 8.86 & 23.01 & 27.49 & -11.58 & -38.36 & -24.11 & -18.97 & -45.66 & -13.29 & -86.32 \\
\hline F1D8Q5 & 8.86 & 23.01 & 27.49 & -11.58 & -38.36 & -24.11 & -18.97 & -45.66 & -13.29 & -86.32 \\
\hline 000429 & 2.49 & 28.04 & 70.37 & 22.06 & 8.36 & -6.83 & -1.25 & 0.17 & -3.31 & -37.47 \\
\hline 014733 & -11.29 & 33.54 & 64.33 & 18.92 & -3.46 & -14.49 & -4.39 & -19.35 & -19.71 & -81.71 \\
\hline 014908 & 7.88 & 10.88 & 1.09 & -26.44 & -56.17 & -37.68 & -27.40 & -37.78 & -25.79 & -40.12 \\
\hline 015169 & -2.32 & 9.61 & 9.52 & 0.25 & -21.32 & -9.90 & -11.25 & -55.27 & -7.43 & -141.66 \\
\hline 043194 & 8.86 & 23.01 & 27.49 & -11.58 & -38.36 & -24.11 & -18.97 & -45.66 & -13.29 & -86.32 \\
\hline 043427 & -6.19 & 17.92 & -28.97 & -12.69 & -45.73 & -35.46 & -21.33 & -26.52 & -24.18 & -38.90 \\
\hline 043623 & 0.02 & 9.39 & -10.51 & -21.77 & -49.67 & -40.38 & -25.11 & -33.10 & -24.30 & -53.26 \\
\hline 060346 & 8.86 & 23.01 & 27.49 & -11.58 & -38.36 & -24.11 & -18.97 & -45.66 & -13.29 & -86.32 \\
\hline 060502 & 8.86 & 23.01 & 27.49 & -11.58 & -38.36 & -24.11 & -18.97 & -45.66 & -13.29 & -86.32 \\
\hline 075165 & 8.86 & 23.01 & 27.49 & -11.58 & -38.36 & -24.11 & -18.97 & -45.66 & -13.29 & -86.32 \\
\hline 075354 & -0.47 & 12.87 & -4.95 & -22.64 & -57.82 & -39.54 & -27.50 & -48.84 & -26.65 & -62.15 \\
\hline 075398 & -3.40 & 4.49 & -41.42 & -19.41 & -40.81 & -35.09 & -19.95 & -50.49 & -26.21 & -47.54 \\
\hline 075448 & 8.86 & 23.01 & 27.49 & -11.58 & -38.36 & -24.11 & -18.97 & -45.66 & -13.29 & -86.32 \\
\hline 075581 & 8.86 & 23.01 & 27.49 & -11.58 & -38.36 & -24.11 & -18.97 & -45.66 & -13.29 & -86.32 \\
\hline 075689 & -4.13 & 29.86 & 6.42 & 17.34 & -24.47 & -14.77 & -13.24 & -37.71 & -26.80 & -79.35 \\
\hline 075925 & 8.86 & 23.01 & 27.49 & -11.58 & -38.36 & -24.11 & -18.97 & -45.66 & -13.29 & -86.32 \\
\hline 075935 & 0.65 & 15.38 & -23.59 & -18.42 & -48.95 & -33.33 & -22.85 & -28.66 & -24.64 & -25.20 \\
\hline 075952 & 8.86 & 23.01 & 27.49 & -11.58 & -38.36 & -24.11 & -18.97 & -45.66 & -13.29 & -86.32 \\
\hline 094805 & 12.99 & 31.73 & 29.31 & 11.31 & -25.36 & -18.45 & -5.37 & -23.77 & -29.10 & -91.22 \\
\hline 095377 & 20.14 & 43.55 & 10.14 & 25.34 & -12.45 & -0.29 & -6.21 & -28.93 & -16.76 & -68.57 \\
\hline 095863 & 2.06 & 16.59 & 0.10 & -20.47 & -42.12 & -36.77 & -24.19 & -46.52 & -17.87 & -91.25 \\
\hline P01106 & 8.86 & 23.01 & 27.49 & -11.58 & -38.36 & -24.11 & -18.97 & -45.66 & -13.29 & -86.32 \\
\hline P01871 & 2.51 & 17.40 & -24.86 & -11.38 & -42.85 & -34.98 & -19.92 & -29.45 & -27.09 & -51.67 \\
\hline P04406 & -1.53 & 24.76 & 46.33 & -6.91 & -39.48 & -24.94 & -18.50 & -40.22 & -28.59 & -128.05 \\
\hline P04637 & 8.99 & 47.04 & 27.71 & 21.99 & -19.08 & -8.96 & -10.57 & -45.67 & -22.47 & -74.29 \\
\hline P05067 & 8.86 & 23.01 & 27.49 & -11.58 & -38.36 & -24.11 & -18.97 & -45.66 & -13.29 & -86.32 \\
\hline P05386 & -0.20 & 32.16 & 40.33 & 16.05 & -9.49 & -12.47 & -3.14 & -20.44 & -22.84 & -73.84 \\
\hline P05412 & -2.51 & 9.71 & -4.48 & -19.00 & -45.38 & -36.80 & -23.54 & -35.13 & -22.08 & -42.58 \\
\hline P05452 & 12.33 & 35.16 & 26.80 & 25.71 & -6.60 & -8.31 & -5.34 & -37.41 & -21.13 & -73.72 \\
\hline P07108 & -5.46 & 27.96 & 50.26 & 4.32 & -20.77 & -15.95 & -7.93 & -26.78 & -23.00 & -79.94 \\
\hline P07199 & 8.86 & 23.01 & 27.49 & -11.58 & -38.36 & -24.11 & -18.97 & -45.66 & -13.29 & -86.32 \\
\hline P08670 & 6.37 & 15.25 & -18.01 & -1.08 & -30.15 & -21.87 & -14.37 & -33.99 & -20.42 & -34.12 \\
\hline P10275 & 8.86 & 23.01 & 27.49 & -11.58 & -38.36 & -24.11 & -18.97 & -45.66 & -13.29 & -86.32 \\
\hline P10636 & 8.86 & 23.01 & 27.49 & -11.58 & -38.36 & -24.11 & -18.97 & -45.66 & -13.29 & -86.32 \\
\hline P13807 & 2.49 & 28.04 & 70.37 & 22.06 & 8.36 & -6.83 & -1.25 & 0.17 & -3.31 & -37.47 \\
\hline P15880 & 7.25 & 27.52 & 30.19 & 20.29 & -12.68 & -15.53 & -6.79 & -34.84 & -26.59 & -105.95 \\
\hline P16220 & 6.28 & 18.69 & -18.71 & -21.84 & -50.44 & -31.23 & -25.78 & -39.56 & -27.59 & -27.71 \\
\hline P16989 & -3.24 & 20.50 & -9.85 & -2.69 & -49.61 & -32.78 & -21.27 & -43.80 & -24.80 & -51.99 \\
\hline
\end{tabular}




\begin{tabular}{|c|c|c|c|c|c|c|c|c|c|c|}
\hline P17252 & -0.12 & 16.75 & 39.22 & -10.17 & -52.44 & -31.26 & -20.46 & -55.60 & -33.67 & -107.25 \\
\hline P17612 & -53.88 & -41.82 & 103.01 & 117.20 & 73.58 & 72.01 & 31.69 & -4.54 & 47.84 & -35.23 \\
\hline P18031 & 7.99 & 16.08 & 32.46 & -16.22 & -52.01 & -31.69 & -19.42 & -56.54 & -31.66 & -132.67 \\
\hline P19793 & 2.49 & 28.04 & 70.37 & 22.06 & 8.36 & -6.83 & -1.25 & 0.17 & -3.31 & -37.47 \\
\hline P19838 & 8.86 & 23.01 & 27.49 & -11.58 & -38.36 & -24.11 & -18.97 & -45.66 & -13.29 & -86.32 \\
\hline P20749 & 9.05 & 38.61 & 28.81 & 20.45 & -17.62 & -11.00 & -8.30 & -45.87 & -20.90 & -63.82 \\
\hline P21246 & 2.20 & 20.68 & -14.48 & -4.68 & -37.20 & -24.47 & -18.45 & -17.16 & -21.05 & -18.19 \\
\hline P23246 & -0.10 & 10.13 & -30.72 & -24.68 & -62.54 & -32.71 & -28.29 & -69.58 & -28.57 & -61.68 \\
\hline P24385 & -11.12 & 15.94 & 32.84 & -8.74 & -47.28 & -26.87 & -21.63 & -49.33 & -27.68 & -96.25 \\
\hline P24864 & -11.60 & 26.57 & 46.55 & 21.75 & -12.55 & -14.59 & -2.85 & -20.77 & -23.38 & -64.16 \\
\hline P25054 & 8.86 & 23.01 & 27.49 & -11.58 & -38.36 & -24.11 & -18.97 & -45.66 & -13.29 & -86.32 \\
\hline P26358 & 2.49 & 28.04 & 70.37 & 22.06 & 8.36 & -6.83 & -1.25 & 0.17 & -3.31 & -37.47 \\
\hline P26641 & 8.86 & 23.01 & 27.49 & -11.58 & -38.36 & -24.11 & -18.97 & -45.66 & -13.29 & -86.32 \\
\hline P27816 & 8.86 & 23.01 & 27.49 & -11.58 & -38.36 & -24.11 & -18.97 & -45.66 & -13.29 & -86.32 \\
\hline P28482 & -3.28 & 29.61 & 58.28 & 9.51 & -14.81 & -19.53 & -7.56 & -27.44 & -23.83 & -69.82 \\
\hline P31749 & -53.88 & -41.82 & 103.01 & 117.20 & 73.58 & 72.01 & 31.69 & -4.54 & 47.84 & -35.23 \\
\hline P31751 & -53.88 & -41.82 & 103.01 & 117.20 & 73.58 & 72.01 & 31.69 & -4.54 & 47.84 & -35.23 \\
\hline P32456 & 2.49 & 28.04 & 70.37 & 22.06 & 8.36 & -6.83 & -1.25 & 0.17 & -3.31 & -37.47 \\
\hline P33076 & 8.86 & 23.01 & 27.49 & -11.58 & -38.36 & -24.11 & -18.97 & -45.66 & -13.29 & -86.32 \\
\hline P33176 & 8.86 & 23.01 & 27.49 & -11.58 & -38.36 & -24.11 & -18.97 & -45.66 & -13.29 & -86.32 \\
\hline P34932 & 2.49 & 28.04 & 70.37 & 22.06 & 8.36 & -6.83 & -1.25 & 0.17 & -3.31 & -37.47 \\
\hline P35222 & 2.49 & 28.04 & 70.37 & 22.06 & 8.36 & -6.83 & -1.25 & 0.17 & -3.31 & -37.47 \\
\hline P36956 & 8.86 & 23.01 & 27.49 & -11.58 & -38.36 & -24.11 & -18.97 & -45.66 & -13.29 & -86.32 \\
\hline P39748 & 7.52 & 26.06 & 18.14 & -4.01 & -41.33 & -31.46 & -9.60 & -46.27 & -33.01 & -118.12 \\
\hline P41236 & 2.92 & 15.57 & -4.88 & -23.62 & -53.31 & -43.07 & -23.44 & -39.86 & -20.94 & -66.61 \\
\hline P42345 & 8.86 & 23.01 & 27.49 & -11.58 & -38.36 & -24.11 & -18.97 & -45.66 & -13.29 & -86.32 \\
\hline P46379 & 8.86 & 23.01 & 27.49 & -11.58 & -38.36 & -24.11 & -18.97 & -45.66 & -13.29 & -86.32 \\
\hline P46531 & 8.86 & 23.01 & 27.49 & -11.58 & -38.36 & -24.11 & -18.97 & -45.66 & -13.29 & -86.32 \\
\hline P47897 & 2.49 & 28.04 & 70.37 & 22.06 & 8.36 & -6.83 & -1.25 & 0.17 & -3.31 & -37.47 \\
\hline P48436 & 8.86 & 23.01 & 27.49 & -11.58 & -38.36 & -24.11 & -18.97 & -45.66 & -13.29 & -86.32 \\
\hline P48740 & 2.49 & 28.04 & 70.37 & 22.06 & 8.36 & -6.83 & -1.25 & 0.17 & -3.31 & -37.47 \\
\hline P49407 & 10.23 & 24.11 & 42.73 & 20.37 & -7.78 & -10.08 & -5.23 & -23.64 & -22.88 & -106.11 \\
\hline P49768 & -3.56 & 23.44 & 34.64 & -0.14 & -52.32 & -24.24 & -16.04 & -42.30 & -26.94 & -116.02 \\
\hline P49815 & 8.86 & 23.01 & 27.49 & -11.58 & -38.36 & -24.11 & -18.97 & -45.66 & -13.29 & -86.32 \\
\hline P49841 & 4.47 & 19.71 & 44.82 & -10.07 & -24.97 & -16.47 & -12.90 & -44.45 & -6.18 & -92.09 \\
\hline P51668 & -10.11 & 26.40 & 54.63 & 8.20 & -24.23 & -19.13 & -9.80 & -31.45 & -17.77 & -77.60 \\
\hline P52742 & 8.86 & 23.01 & 27.49 & -11.58 & -38.36 & -24.11 & -18.97 & -45.66 & -13.29 & -86.32 \\
\hline P54252 & 5.32 & 30.37 & 43.10 & -1.66 & -30.11 & -22.06 & -9.77 & -36.13 & -27.70 & -89.41 \\
\hline P56524 & 8.86 & 23.01 & 27.49 & -11.58 & -38.36 & -24.11 & -18.97 & -45.66 & -13.29 & -86.32 \\
\hline P56693 & 4.95 & 20.17 & -16.04 & 0.29 & -41.07 & -24.13 & -15.92 & -42.03 & -22.62 & -42.54 \\
\hline P59665 & 8.86 & 23.01 & 27.49 & -11.58 & -38.36 & -24.11 & -18.97 & -45.66 & -13.29 & -86.32 \\
\hline P61960 & -7.04 & 31.69 & 47.25 & 9.04 & -25.24 & -22.39 & -9.84 & -33.80 & -26.73 & -83.55 \\
\hline P61968 & -3.71 & 10.49 & -8.90 & -20.13 & -49.00 & -41.40 & -23.81 & -33.16 & -22.55 & -60.29 \\
\hline P62879 & 1.36 & 14.29 & -34.90 & -6.02 & -45.40 & -32.62 & -21.26 & -24.35 & -25.27 & -27.95 \\
\hline P67809 & -0.34 & 15.82 & -22.72 & -17.62 & -34.33 & -36.32 & -19.14 & -17.34 & -26.73 & -13.95 \\
\hline P67870 & -8.01 & 29.45 & 32.59 & 6.20 & -32.12 & -24.78 & -13.40 & -43.76 & -30.95 & -100.04 \\
\hline
\end{tabular}




\begin{tabular}{|c|c|c|c|c|c|c|c|c|c|c|}
\hline P68104 & 2.49 & 28.04 & 70.37 & 22.06 & 8.36 & -6.83 & -1.25 & 0.17 & -3.31 & -37.47 \\
\hline P78527 & 8.86 & 23.01 & 27.49 & -11.58 & -38.36 & -24.11 & -18.97 & -45.66 & -13.29 & -86.32 \\
\hline Q00013 & -3.90 & 17.60 & -25.76 & -17.24 & -52.44 & -35.48 & -24.86 & -28.97 & -26.32 & -42.62 \\
\hline Q00987 & 11.27 & 24.65 & -12.13 & -1.92 & -41.45 & -23.79 & -18.89 & -34.10 & -19.60 & -30.59 \\
\hline Q01201 & -10.89 & 22.70 & 54.98 & 11.08 & -22.62 & -15.13 & -11.95 & -34.12 & -21.50 & -84.83 \\
\hline Q03701 & 8.86 & 23.01 & 27.49 & -11.58 & -38.36 & -24.11 & -18.97 & -45.66 & -13.29 & -86.32 \\
\hline Q04721 & 8.86 & 23.01 & 27.49 & -11.58 & -38.36 & -24.11 & -18.97 & -45.66 & -13.29 & -86.32 \\
\hline Q04724 & 8.86 & 23.01 & 27.49 & -11.58 & -38.36 & -24.11 & -18.97 & -45.66 & -13.29 & -86.32 \\
\hline Q06124 & 9.08 & 23.84 & 34.31 & -3.11 & -27.37 & -21.18 & -10.01 & -26.28 & -26.52 & -110.49 \\
\hline Q06330 & 2.49 & 28.04 & 70.37 & 22.06 & 8.36 & -6.83 & -1.25 & 0.17 & -3.31 & -37.47 \\
\hline Q07812 & 3.29 & 23.26 & 29.67 & 10.00 & -26.68 & -18.45 & -11.78 & -42.67 & -28.85 & -107.91 \\
\hline Q08499 & 2.49 & 28.04 & 70.37 & 22.06 & 8.36 & -6.83 & -1.25 & 0.17 & -3.31 & -37.47 \\
\hline Q12873 & 8.86 & 23.01 & 27.49 & -11.58 & -38.36 & -24.11 & -18.97 & -45.66 & -13.29 & -86.32 \\
\hline Q13144 & 8.86 & 23.01 & 27.49 & -11.58 & -38.36 & -24.11 & -18.97 & -45.66 & -13.29 & -86.32 \\
\hline Q13239 & 13.91 & 48.77 & 24.04 & 32.84 & 4.65 & 2.25 & -2.53 & -20.10 & -15.44 & -81.29 \\
\hline Q13467 & -1.29 & 18.28 & -17.54 & -8.71 & -40.56 & -30.03 & -19.59 & -31.55 & -18.39 & -27.76 \\
\hline Q13541 & -0.23 & 9.26 & -11.77 & -21.82 & -47.74 & -40.63 & -23.11 & -39.80 & -21.11 & -52.08 \\
\hline Q13794 & 8.86 & 23.01 & 27.49 & -11.58 & -38.36 & -24.11 & -18.97 & -45.66 & -13.29 & -86.32 \\
\hline Q13887 & 0.63 & 16.04 & -21.54 & -1.23 & -40.23 & -29.55 & -20.17 & -44.24 & -20.93 & -41.67 \\
\hline Q14103 & 1.65 & 15.21 & -19.62 & -10.80 & -44.81 & -28.06 & -20.60 & -46.57 & -24.22 & -51.45 \\
\hline Q14147 & 8.86 & 23.01 & 27.49 & -11.58 & -38.36 & -24.11 & -18.97 & -45.66 & -13.29 & -86.32 \\
\hline Q14596 & 8.86 & 23.01 & 27.49 & -11.58 & -38.36 & -24.11 & -18.97 & -45.66 & -13.29 & -86.32 \\
\hline Q15029 & 8.86 & 23.01 & 27.49 & -11.58 & -38.36 & -24.11 & -18.97 & -45.66 & -13.29 & -86.32 \\
\hline Q15404 & -9.76 & 23.67 & 30.67 & -5.67 & -40.42 & -27.54 & -14.12 & -39.11 & -28.39 & -120.38 \\
\hline Q15418 & 8.86 & 23.01 & 27.49 & -11.58 & -38.36 & -24.11 & -18.97 & -45.66 & -13.29 & -86.32 \\
\hline Q15797 & -1.54 & 17.50 & -28.60 & -19.59 & -50.87 & -35.05 & -24.51 & -35.65 & -24.27 & -46.47 \\
\hline Q15828 & 9.19 & 34.68 & 3.34 & 10.39 & -27.35 & -12.04 & -14.49 & -57.00 & -23.42 & -83.66 \\
\hline Q15847 & 8.86 & 23.01 & 27.49 & -11.58 & -38.36 & -24.11 & -18.97 & -45.66 & -13.29 & -86.32 \\
\hline Q16236 & -4.74 & 11.31 & -19.82 & -21.73 & -44.16 & -30.79 & -25.44 & -41.44 & -29.46 & -37.36 \\
\hline Q16635 & 4.81 & 12.58 & -20.50 & -8.18 & -48.54 & -38.81 & -21.32 & -42.21 & -20.87 & -78.48 \\
\hline Q16877 & 2.49 & 28.04 & 70.37 & 22.06 & 8.36 & -6.83 & -1.25 & 0.17 & -3.31 & -37.47 \\
\hline Q2T9K0 & 8.86 & 23.01 & 27.49 & -11.58 & -38.36 & -24.11 & -18.97 & -45.66 & -13.29 & -86.32 \\
\hline Q53GA5 & -9.71 & 16.13 & 0.27 & -13.62 & -44.12 & -26.92 & -24.45 & -47.16 & -24.17 & -31.23 \\
\hline Q59EF7 & 8.86 & 23.01 & 27.49 & -11.58 & -38.36 & -24.11 & -18.97 & -45.66 & -13.29 & -86.32 \\
\hline Q59FP7 & 8.86 & 23.01 & 27.49 & -11.58 & -38.36 & -24.11 & -18.97 & -45.66 & -13.29 & -86.32 \\
\hline Q5H9Q5 & 7.90 & 19.07 & -11.99 & -22.96 & -58.89 & -33.47 & -26.12 & -33.17 & -28.72 & -13.83 \\
\hline Q5S007 & 8.86 & 23.01 & 27.49 & -11.58 & -38.36 & -24.11 & -18.97 & -45.66 & -13.29 & -86.32 \\
\hline Q5TBU5 & 2.49 & 28.04 & 70.37 & 22.06 & 8.36 & -6.83 & -1.25 & 0.17 & -3.31 & -37.47 \\
\hline Q66X48 & 8.86 & 23.01 & 27.49 & -11.58 & -38.36 & -24.11 & -18.97 & -45.66 & -13.29 & -86.32 \\
\hline Q68CJ9 & 13.92 & 30.41 & -13.38 & 14.16 & -35.19 & -18.12 & -14.92 & -29.18 & -20.66 & -35.28 \\
\hline Q6NUN9 & 8.86 & 23.01 & 27.49 & -11.58 & -38.36 & -24.11 & -18.97 & -45.66 & -13.29 & -86.32 \\
\hline Q6R327 & 8.86 & 23.01 & 27.49 & -11.58 & -38.36 & -24.11 & -18.97 & -45.66 & -13.29 & -86.32 \\
\hline Q6UXH1 & -6.46 & 5.91 & -13.62 & -14.74 & -46.50 & -38.76 & -21.32 & -34.14 & -21.33 & -60.93 \\
\hline Q71U36 & -18.43 & 25.67 & 59.10 & 15.89 & -12.08 & -19.47 & -6.23 & -26.42 & -24.18 & -59.30 \\
\hline Q75N90 & 8.86 & 23.01 & 27.49 & -11.58 & -38.36 & -24.11 & -18.97 & -45.66 & -13.29 & -86.32 \\
\hline Q7L266 & -4.19 & 27.18 & 46.82 & -0.33 & -28.19 & -20.17 & -14.45 & -27.57 & -25.06 & -88.62 \\
\hline
\end{tabular}




\begin{tabular}{|c|c|c|c|c|c|c|c|c|c|c|}
\hline Q7TQJ8 & 8.86 & 23.01 & 27.49 & -11.58 & -38.36 & -24.11 & -18.97 & -45.66 & -13.29 & -86.32 \\
\hline Q7Z5J4 & 8.86 & 23.01 & 27.49 & -11.58 & -38.36 & -24.11 & -18.97 & -45.66 & -13.29 & -86.32 \\
\hline Q86SX1 & -1.50 & 8.90 & -36.35 & -30.35 & -49.31 & -42.90 & -24.78 & -35.77 & -31.56 & -18.85 \\
\hline Q86TP1 & -0.11 & 9.31 & -35.17 & -27.50 & -66.76 & -45.55 & -29.72 & -55.58 & -30.39 & -32.73 \\
\hline Q86WZ6 & 8.86 & 23.01 & 27.49 & -11.58 & -38.36 & -24.11 & -18.97 & -45.66 & -13.29 & -86.32 \\
\hline Q8IWV7 & 8.86 & 23.01 & 27.49 & -11.58 & -38.36 & -24.11 & -18.97 & -45.66 & -13.29 & -86.32 \\
\hline Q8IX07 & 8.86 & 23.01 & 27.49 & -11.58 & -38.36 & -24.11 & -18.97 & -45.66 & -13.29 & -86.32 \\
\hline Q8N205 & 8.86 & 23.01 & 27.49 & -11.58 & -38.36 & -24.11 & -18.97 & -45.66 & -13.29 & -86.32 \\
\hline Q8N4C6 & 8.86 & 23.01 & 27.49 & -11.58 & -38.36 & -24.11 & -18.97 & -45.66 & -13.29 & -86.32 \\
\hline Q8NF22 & -4.57 & 17.95 & -11.71 & -2.46 & -39.66 & -30.84 & -21.07 & -50.31 & -21.75 & -54.40 \\
\hline Q8NF37 & -4.89 & 14.90 & -30.40 & -15.24 & -51.02 & -30.93 & -23.56 & -30.71 & -26.17 & -55.03 \\
\hline Q8TDD2 & -1.63 & 25.93 & -13.57 & -12.83 & -42.45 & -28.68 & -19.50 & -38.67 & -22.87 & -42.11 \\
\hline Q8TDN6 & -5.34 & 8.81 & -14.19 & -9.27 & -40.70 & -33.72 & -17.34 & -32.57 & -14.64 & -64.93 \\
\hline Q8TDZ2 & 8.86 & 23.01 & 27.49 & -11.58 & -38.36 & -24.11 & -18.97 & -45.66 & -13.29 & -86.32 \\
\hline Q92837 & -11.09 & 1.16 & -7.16 & -5.62 & -38.02 & -12.23 & -15.26 & -45.48 & -23.27 & -188.79 \\
\hline Q92945 & 8.86 & 23.01 & 27.49 & -11.58 & -38.36 & -24.11 & -18.97 & -45.66 & -13.29 & -86.32 \\
\hline Q969Q0 & -2.87 & 24.05 & 42.20 & 11.03 & -17.75 & -19.28 & -10.16 & -29.74 & -21.95 & -74.78 \\
\hline Q96A73 & -0.11 & 16.42 & -21.35 & -2.57 & -41.28 & -29.02 & -20.35 & -44.17 & -22.20 & -49.44 \\
\hline Q96BR1 & -53.88 & -41.82 & 103.01 & 117.20 & 73.58 & 72.01 & 31.69 & -4.54 & 47.84 & -35.23 \\
\hline Q96G01 & 8.86 & 23.01 & 27.49 & -11.58 & -38.36 & -24.11 & -18.97 & -45.66 & -13.29 & -86.32 \\
\hline Q96GR2 & 8.86 & 23.01 & 27.49 & -11.58 & -38.36 & -24.11 & -18.97 & -45.66 & -13.29 & -86.32 \\
\hline Q96HA7 & 8.86 & 23.01 & 27.49 & -11.58 & -38.36 & -24.11 & -18.97 & -45.66 & -13.29 & -86.32 \\
\hline Q96ID5 & -0.28 & 21.93 & -19.88 & -8.86 & -50.14 & -36.88 & -23.39 & -30.32 & -25.09 & -26.92 \\
\hline Q96PV7 & 8.86 & 23.01 & 27.49 & -11.58 & -38.36 & -24.11 & -18.97 & -45.66 & -13.29 & -86.32 \\
\hline Q99502 & 12.18 & 20.03 & -11.16 & -15.41 & -56.58 & -34.38 & -24.65 & -46.21 & -33.35 & -32.91 \\
\hline Q99956 & 8.86 & 23.01 & 27.49 & -11.58 & -38.36 & -24.11 & -18.97 & -45.66 & -13.29 & -86.32 \\
\hline Q9BTE0 & 4.53 & 41.90 & 57.36 & 25.49 & 1.40 & -10.89 & -0.22 & 0.47 & -20.53 & -75.54 \\
\hline Q9BZV1 & -8.68 & 18.99 & -20.54 & -17.74 & -52.22 & -37.63 & -23.05 & -40.23 & -25.69 & -51.21 \\
\hline Q9C0J9 & 1.74 & 14.96 & -23.51 & -14.70 & -51.35 & -28.64 & -25.48 & -57.83 & -28.05 & -55.66 \\
\hline Q9H095 & -6.35 & 18.50 & -28.24 & -21.52 & -56.07 & -40.77 & -25.61 & -29.21 & -27.70 & -17.29 \\
\hline Q9H0C8 & -4.87 & 28.06 & 58.64 & 25.45 & -1.45 & -12.83 & -4.00 & -14.56 & -16.78 & -56.62 \\
\hline Q9H1J1 & 8.86 & 23.01 & 27.49 & -11.58 & -38.36 & -24.11 & -18.97 & -45.66 & -13.29 & -86.32 \\
\hline Q9H254 & 8.86 & 23.01 & 27.49 & -11.58 & -38.36 & -24.11 & -18.97 & -45.66 & -13.29 & -86.32 \\
\hline Q9H4H8 & 8.63 & 26.10 & -13.93 & 15.48 & -31.70 & -20.08 & -13.23 & -22.20 & -18.42 & -34.80 \\
\hline Q9H5C8 & -1.58 & 12.78 & -28.17 & -24.43 & -63.58 & -37.14 & -26.87 & -41.09 & -31.72 & -45.77 \\
\hline Q9HBH7 & 2.49 & 28.04 & 70.37 & 22.06 & 8.36 & -6.83 & -1.25 & 0.17 & -3.31 & -37.47 \\
\hline Q9NPA3 & -7.56 & 14.79 & -17.12 & -11.00 & -43.46 & -32.45 & -20.36 & -32.17 & -22.99 & -24.92 \\
\hline Q9NQW7 & 2.49 & 28.04 & 70.37 & 22.06 & 8.36 & -6.83 & -1.25 & 0.17 & -3.31 & -37.47 \\
\hline Q9NWM8 & 6.07 & 18.93 & 2.68 & -1.59 & -42.67 & -21.17 & -12.67 & -39.85 & -32.70 & -104.71 \\
\hline Q9NX09 & -15.76 & 40.64 & 42.25 & 23.78 & -0.51 & -9.10 & -3.98 & -9.91 & -20.92 & -44.48 \\
\hline Q9P1W9 & 25.76 & 77.74 & 63.72 & 140.53 & 79.93 & 39.40 & 31.18 & 12.59 & 30.50 & -7.00 \\
\hline Q9P1Y5 & 8.86 & 23.01 & 27.49 & -11.58 & -38.36 & -24.11 & -18.97 & -45.66 & -13.29 & -86.32 \\
\hline Q9P2X7 & 8.86 & 23.01 & 27.49 & -11.58 & -38.36 & -24.11 & -18.97 & -45.66 & -13.29 & -86.32 \\
\hline Q9UBK2 & 8.86 & 23.01 & 27.49 & -11.58 & -38.36 & -24.11 & -18.97 & -45.66 & -13.29 & -86.32 \\
\hline Q9UFD5 & -3.80 & 15.74 & -20.75 & 0.14 & -39.51 & -25.80 & -18.70 & -55.33 & -21.45 & -68.99 \\
\hline Q9UHR5 & -6.01 & 14.63 & -26.32 & -20.07 & -42.56 & -37.18 & -24.10 & -39.64 & -28.53 & -42.24 \\
\hline
\end{tabular}




\begin{tabular}{|c|c|c|c|c|c|c|c|c|c|c|}
\hline Q9UHY1 & 8.86 & 23.01 & 27.49 & -11.58 & -38.36 & -24.11 & -18.97 & -45.66 & -13.29 & -86.32 \\
\hline Q9UID3 & 8.86 & 23.01 & 27.49 & -11.58 & -38.36 & -24.11 & -18.97 & -45.66 & -13.29 & -86.32 \\
\hline Q9UII2 & -5.86 & 30.36 & 53.70 & 10.96 & -9.43 & -16.07 & -4.54 & -11.92 & -21.27 & -81.44 \\
\hline Q9UJW3 & 17.08 & 27.83 & -5.13 & 10.66 & -35.81 & -11.71 & -11.87 & -36.71 & -35.33 & -165.19 \\
\hline Q9UKA4 & 8.86 & 23.01 & 27.49 & -11.58 & -38.36 & -24.11 & -18.97 & -45.66 & -13.29 & -86.32 \\
\hline Q9UKB1 & 1.74 & 28.43 & 47.16 & 6.42 & -15.04 & -20.57 & -7.22 & -18.88 & -24.83 & -85.59 \\
\hline Q9UKR5 & 3.30 & 23.62 & -15.68 & -16.55 & -62.49 & -37.18 & -25.29 & -40.92 & -26.70 & -51.93 \\
\hline Q9UKY1 & 8.86 & 23.01 & 27.49 & -11.58 & -38.36 & -24.11 & -18.97 & -45.66 & -13.29 & -86.32 \\
\hline Q9UNZ2 & -1.70 & 9.21 & -11.73 & -21.54 & -49.05 & -43.06 & -23.71 & -35.00 & -24.49 & -56.66 \\
\hline Q9UP38 & -10.41 & 19.38 & -27.62 & -19.32 & -51.68 & -38.63 & -24.37 & -36.46 & -27.77 & -38.87 \\
\hline Q9Y243 & 3.57 & 20.87 & 47.42 & 12.35 & -20.80 & -20.17 & -6.82 & -29.08 & -27.70 & -95.35 \\
\hline Q9Y297 & -10.18 & 26.24 & 55.84 & 18.86 & -9.87 & -14.89 & -4.39 & -19.39 & -17.19 & -54.06 \\
\hline Q9Y2T1 & 8.86 & 23.01 & 27.49 & -11.58 & -38.36 & -24.11 & -18.97 & -45.66 & -13.29 & -86.32 \\
\hline Q9Y383 & -4.72 & 9.99 & -9.39 & -19.03 & -46.45 & -40.16 & -22.28 & -32.81 & -21.68 & -50.64 \\
\hline Q9Y483 & 8.86 & 23.01 & 27.49 & -11.58 & -38.36 & -24.11 & -18.97 & -45.66 & -13.29 & -86.32 \\
\hline Q9Y4A8 & -1.89 & 21.83 & -5.98 & 4.95 & -33.96 & -18.59 & -16.45 & -49.25 & -21.00 & -55.60 \\
\hline Q9Y4X0 & -5.50 & 10.16 & -8.92 & -18.04 & -47.65 & -41.13 & -20.92 & -32.66 & -22.75 & -55.84 \\
\hline Q9Y5W3 & 8.24 & 21.06 & -11.54 & 10.70 & -35.33 & -24.00 & -16.51 & -34.76 & -19.07 & -26.69 \\
\hline Q9Y600 & 11.69 & 25.16 & 29.96 & 17.58 & -14.07 & -11.45 & -7.99 & -26.79 & -23.36 & -97.00 \\
\hline Q9Y6E2 & 4.26 & 22.29 & -23.28 & -26.28 & -59.29 & -39.22 & -27.88 & -39.09 & -28.24 & -64.14 \\
\hline Q9Y6H5 & 8.86 & 23.01 & 27.49 & -11.58 & -38.36 & -24.11 & -18.97 & -45.66 & -13.29 & -86.32 \\
\hline Q9Y617 & 0.49 & 17.81 & -20.07 & -21.28 & -59.64 & -33.76 & -27.24 & -47.63 & -30.07 & -29.73 \\
\hline Q9Y6K9 & 8.86 & 23.01 & 27.49 & -11.58 & -38.36 & -24.11 & -18.97 & -45.66 & -13.29 & -86.32 \\
\hline Q9Y6Q9 & 8.86 & 23.01 & 27.49 & -11.58 & -38.36 & -24.11 & -18.97 & -45.66 & -13.29 & -86.32 \\
\hline Q9Y6R4 & 8.86 & 23.01 & 27.49 & -11.58 & -38.36 & -24.11 & -18.97 & -45.66 & -13.29 & -86.32 \\
\hline V9HVZ4 & -1.53 & 24.76 & 46.33 & -6.91 & -39.48 & -24.94 & -18.50 & -40.22 & -28.59 & -128.05 \\
\hline V9HW29 & 8.86 & 23.01 & 27.49 & -11.58 & -38.36 & -24.11 & -18.97 & -45.66 & -13.29 & -86.32 \\
\hline V9HWE1 & 6.37 & 15.25 & -18.01 & -1.08 & -30.15 & -21.87 & -14.37 & -33.99 & -20.42 & -34.12 \\
\hline V9HWF0 & -8.30 & 31.33 & 44.68 & 6.06 & -32.97 & -24.75 & -11.36 & -39.69 & -30.06 & -106.24 \\
\hline
\end{tabular}

The UniProt ID (UI) related to each specific GSK-3 $\beta$ partner and the comparison between affinities in LiCl- and NaCl-containing environments ( $z$ scores) for set 1 along MD trajectory segments are depicted. The segments are denoted as in Figure 1. Colors in $z$ score columns are indicative of changes in affinity. Blue and orange indicate a lower and a higher affinity in the presence of $\mathrm{LiCl}$ than in the presence of $\mathrm{NaCl}$, respectively. Gray indicates no significant difference between affinities in the two environments. 
Table S4. Number of GSK-3 $\beta$ PBPs with lower, equal, and higher affinities in the presence of $\mathrm{LiCl}$ than those in the presence of $\mathrm{NaCl}$ in each pathway.

\begin{tabular}{|c|c|c|c|c|c|}
\hline $\begin{array}{l}\text { Pathway short } \\
\text { name }\end{array}$ & Pathway & All & $\begin{array}{l}\text { Lower } \\
\text { affinity }\end{array}$ & $\begin{array}{l}\text { Equal } \\
\text { affinity }\end{array}$ & $\begin{array}{l}\text { Higher } \\
\text { affinity }\end{array}$ \\
\hline Wnt & Wnt signaling pathway & 19 & 4 & 1 & 14 \\
\hline Angiogenesis & Angiogenesis & 17 & 5 & 1 & 11 \\
\hline GRHR & Gonadotropin-releasing hormone receptor pathway & 15 & 5 & 0 & 10 \\
\hline CCKR & CCKR signaling map & 15 & 4 & 0 & 11 \\
\hline Apoptosis & Apoptosis signaling pathway & 12 & 4 & 3 & 5 \\
\hline Inflammation & Inflammation mediated by chemokine and cytokine signaling pathway & 11 & 5 & 3 & 3 \\
\hline Alz presenilin & Alzheimer disease-presenilin pathway & 10 & 2 & 0 & 8 \\
\hline PDGF & PDGF signaling pathway & 10 & 2 & 0 & 8 \\
\hline Interleukin & Interleukin signaling pathway & 9 & 3 & 1 & 5 \\
\hline p53 & p53 pathway & 8 & 3 & 2 & 3 \\
\hline p53 loops2 & p53 pathway feedback loops 2 & 8 & 4 & 2 & 2 \\
\hline Ras & Ras Pathway & 8 & 3 & 1 & 4 \\
\hline Huntington & Huntington disease & 8 & 3 & 2 & 3 \\
\hline FGF & FGF signaling pathway & 8 & 4 & 1 & 3 \\
\hline p53 glucose & p53 pathway by glucose deprivation & 7 & 2 & 2 & 3 \\
\hline Gi Gs & Heterotrimeric G-protein signaling pathway-Gi alpha and Gs alpha mediated pathway & 7 & 3 & 0 & 4 \\
\hline EGFR & EGF receptor signaling pathway & 7 & 4 & 1 & 2 \\
\hline T cell & $\mathrm{T}$ cell activation & 6 & 3 & 1 & 2 \\
\hline PI3K & PI3 kinase pathway & 6 & 2 & 1 & 3 \\
\hline Endothelin & Endothelin signaling pathway & 6 & 4 & 1 & 1 \\
\hline Cadherin & Cadherin signaling pathway & 6 & 1 & 0 & 5 \\
\hline Parkinson & Parkinson disease & 5 & 3 & 0 & 2 \\
\hline Insulin PKB & Insulin/IGF pathway-protein kinase B signaling cascade & 5 & 2 & 0 & 3 \\
\hline Alz secretase & Alzheimer disease-amyloid secretase pathway & 4 & 1 & 0 & 3 \\
\hline Oxidative & Oxidative stress response & 4 & 0 & 0 & 4 \\
\hline Notch & Notch signaling pathway & 4 & 1 & 0 & 3 \\
\hline Hypoxia & Hypoxia response via HIF activation & 4 & 2 & 1 & 1 \\
\hline Angiotensin & Angiotensin II-stimulated signaling through $\mathrm{G}$ proteins and beta-arrestin & 4 & 2 & 0 & 2 \\
\hline B cell & B cell activation & 4 & 1 & 0 & 3 \\
\hline VEGF & VEGF signaling pathway & 3 & 2 & 0 & 1 \\
\hline Toll receptor & Toll receptor signaling pathway & 3 & 1 & 0 & 2 \\
\hline TGF-beta & TGF-beta signaling pathway & 3 & 1 & 0 & 2 \\
\hline Interferon & Interferon-gamma signaling pathway & 3 & 1 & 0 & 2 \\
\hline Integrin & Integrin signalling pathway & 3 & 1 & 0 & 2 \\
\hline p38 & p38 MAPK pathway & 3 & 0 & 0 & 3 \\
\hline Hedgehog & Hedgehog signaling pathway & 3 & 2 & 0 & 1 \\
\hline Enkephalin & Enkephalin release & 3 & 1 & 0 & 2 \\
\hline FAS & FAS signaling pathway & 3 & 2 & 0 & 1 \\
\hline Ubiquitin & Ubiquitin proteasome pathway & 2 & 0 & 1 & 1 \\
\hline bZIP & Transcription regulation by bZIP transcription factor & 2 & 0 & 0 & 2 \\
\hline Muscarinic 24 & Muscarinic acetylcholine receptor 2 and 4 signaling pathway & 2 & 1 & 0 & 1 \\
\hline Muscarinic 13 & Muscarinic acetylcholine receptor 1 and 3 signaling pathway & 2 & 0 & 0 & 2 \\
\hline Metabotropic II & Metabotropic glutamate receptor group II pathway & 2 & 1 & 0 & 1 \\
\hline GABA receptor & GABA-B receptor II signaling & 2 & 1 & 0 & 1 \\
\hline Metabotropic III & Metabotropic glutamate receptor group III pathway & 2 & 1 & 0 & 1 \\
\hline Insulin MAPK & Insulin/IGF pathway-mitogen activated protein kinase kinase/MAP kinase cascade & 2 & 1 & 0 & 1 \\
\hline TRHR & Thyrotropin-releasing hormone receptor signaling pathway & 2 & 0 & 0 & 2 \\
\hline p53 loops1 & P53 pathway feedback loops 1 & 2 & 0 & 1 & 1 \\
\hline Oxytocin receptor & Oxytocin receptor mediated signaling pathway & 2 & 0 & 0 & 2 \\
\hline Phototransduction & Heterotrimeric G-protein signaling pathway-rod outer segment phototransduction & 2 & 1 & 0 & 1 \\
\hline Gq Go & Heterotrimeric G-protein signaling pathway-Gq alpha and Go alpha mediated pathway & 2 & 0 & 0 & 2 \\
\hline Dopamine receptor & Dopamine receptor mediated signaling pathway & 2 & 1 & 0 & 1 \\
\hline $\mathrm{H} 2 \mathrm{R}$ & Histamine $\mathrm{H} 2$ receptor mediated signaling pathway & 2 & 1 & 0 & 1 \\
\hline H1R & Histamine $\mathrm{H} 1$ receptor mediated signaling pathway & 2 & 0 & 0 & 2 \\
\hline Cell cycle & Cell cycle & 2 & 1 & 0 & 1 \\
\hline Adrenergic beta2 & Beta2 adrenergic receptor signaling pathway & 2 & 1 & 0 & 1 \\
\hline Adrenergic beta1 & Beta 1 adrenergic receptor signaling pathway & 2 & 1 & 0 & 1 \\
\hline $5 \mathrm{HT} 2$ & 5HT2 type receptor mediated signaling pathway & 2 & 0 & 0 & 2 \\
\hline $5 \mathrm{HT} 1$ & $5 \mathrm{HT} 1$ type receptor mediated signaling pathway & 2 & 1 & 0 & 1 \\
\hline Adrenergic alpha & Alpha adrenergic receptor signaling pathway & 1 & 0 & 0 & 1 \\
\hline Heme & Heme biosynthesis & 1 & 1 & 0 & 0 \\
\hline Metabotropic I & Metabotropic glutamate receptor group I pathway & 1 & 1 & 0 & 0 \\
\hline Cannabinoid & Endogenous cannabinoid signaling & 1 & 0 & 0 & 1 \\
\hline JAK/STAT & JAK/STAT signaling pathway & 1 & 0 & 0 & 1 \\
\hline Vitamin D & Vitamin D metabolism and pathway & 1 & 1 & 0 & 0 \\
\hline Proopiomelanocortin & Opioid proopiomelanocortin pathway & 1 & 0 & 0 & 1 \\
\hline Prodynorphin & Opioid prodynorphin pathway & 1 & 0 & 0 & 1 \\
\hline Glycolysis & Glycolysis & 1 & 0 & 0 & 1 \\
\hline Proenkephalin & Opioid proenkephalin pathway & 1 & 0 & 0 & 1 \\
\hline Nicotine & Nicotine pharmacodynamics pathway & 1 & 1 & 0 & 0 \\
\hline GABA & Gamma-aminobutyric acid synthesis & 1 & 0 & 1 & 0 \\
\hline CRFR & Cortocotropin releasing factor receptor signaling pathway & 1 & 0 & 0 & 1 \\
\hline Adrenergic beta3 & Beta3 adrenergic receptor signaling pathway & 1 & 0 & 0 & 1 \\
\hline Coagulation & Blood coagulation & 1 & 0 & 0 & 1 \\
\hline $5 \mathrm{HT} 4$ & 5HT4 type receptor mediated signaling pathway & 1 & 0 & 0 & 1 \\
\hline
\end{tabular}


